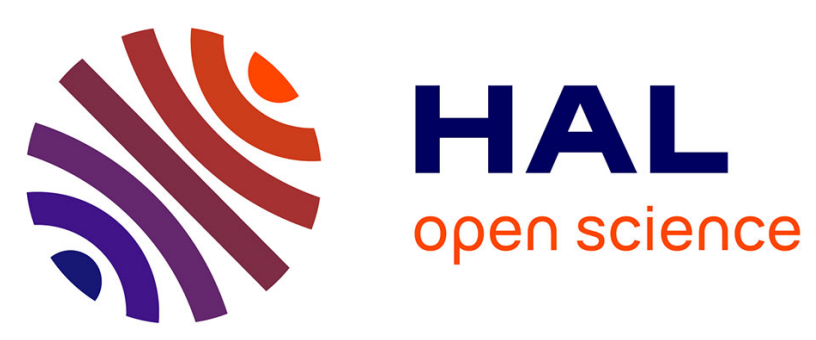

\title{
A continuous material law for modeling thin-sheet piles and their frictional connection
}

\author{
A.S. Pechstein, L.G. Aigner, J. Gerstmayr
}

\section{To cite this version:}

A.S. Pechstein, L.G. Aigner, J. Gerstmayr. A continuous material law for modeling thin-sheet piles and their frictional connection. European Journal of Mechanics - A/Solids, 2011, 30 (5), pp.684. 10.1016/j.euromechsol.2011.04.012 . hal-00769683

\section{HAL Id: hal-00769683 \\ https://hal.science/hal-00769683}

Submitted on 3 Jan 2013

HAL is a multi-disciplinary open access archive for the deposit and dissemination of scientific research documents, whether they are published or not. The documents may come from teaching and research institutions in France or abroad, or from public or private research centers.
L'archive ouverte pluridisciplinaire HAL, est destinée au dépôt et à la diffusion de documents scientifiques de niveau recherche, publiés ou non, émanant des établissements d'enseignement et de recherche français ou étrangers, des laboratoires publics ou privés. 


\section{Accepted Manuscript}

Title: A continuous material law for modeling thin-sheet piles and their frictional connection

Authors: A.S. Pechstein, L.G. Aigner, J. Gerstmayr

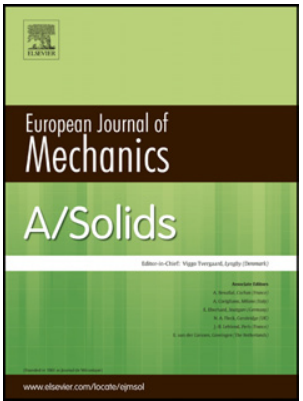

PII:

S0997-7538(11)00058-1

DOI:

10.1016/j.euromechsol.2011.04.012

Reference: EJMSOL 2710

To appear in: European Journal of Mechanics / A Solids

Received Date: 8 July 2010

Revised Date: 18 March 2011

Accepted Date: 26 April 2011

Please cite this article as: Pechstein, A.S., Aigner, L.G., Gerstmayr, J. A continuous material law for modeling thin-sheet piles and their frictional connection, European Journal of Mechanics / A Solids (2011), doi: 10.1016/j.euromechsol.2011.04.012

This is a PDF file of an unedited manuscript that has been accepted for publication. As a service to our customers we are providing this early version of the manuscript. The manuscript will undergo copyediting, typesetting, and review of the resulting proof before it is published in its final form. Please note that during the production process errors may be discovered which could affect the content, and all legal disclaimers that apply to the journal pertain. 


\title{
A continuous material law for modeling thin-sheet piles and their frictional connection
}

\author{
A.S. Pechstein ${ }^{a, *}$, L.G. Aigner ${ }^{\mathrm{b}}$, J. Gerstmayr ${ }^{\mathrm{b}}$ \\ ${ }^{a}$ Institute of Technical Mechanics, Johannes Kepler University Linz, Altenbergerstr. 69, \\ 4040 Linz, Austria \\ ${ }^{b}$ Linz Center of Mechatronics GmbH, Altenbergerstr. 69, 4040 Linz, Austria
}

\begin{abstract}
In the present paper, the mechanical modeling and the numerical simulation of a pile of thin sheets under compressive and in-plane forces is presented. These sheets are not glued or laminated, but interact through frictional contact only. In applications, as for example the core of a large power transformer, such piles may consist of thousands of sheets, which are of thickness below one millimeter, while the dimensions of the pile reaches several meters. Also, several piles may interact by a frictional connection. Such connections are realized by regions where sheets from both stacks overlap mutually. Simulations using a properly meshed original geometry and standard finite element models lead to billions of unknowns for industrial applications. Additionally, the system is highly nonlinear due to the heavily coupled contact conditions posed on thousands of interfaces. Simulations become extremely expensive in terms of both memory and computation time, if not even unsolvable due to numerical convergence problems. The aim of this paper is to present a macroscopic material model, which can be applied to an equivalent homogenized computational domain representing the interconnected sheet piles. An extension of the material law in regions of mutual overlapping due to frictional connections is provided. When using the present approach, the homogenized computational domain can be discretized by a far smaller number of unknowns, while a good overall accuracy is retained. The numerical solution of standardized test problems is presented and verified against analytical considerations.
\end{abstract}

Keywords: thin sheets, homogenization, micro-macro approach, contact, friction, continuum mechanics.

\section{Introduction}

The aim of the present paper is the derivation of a phenomenologically motivated, macroscopic material law for the computational simulation of piles of thin

\footnotetext{
${ }^{*}$ Corresponding author
} 
sheets. This material law allows to treat the pile as one equivalent geometrical solid body. Such a simplification of the geometry leads to reduced models, which are sufficiently accurate for the computational analysis of stability properties. Utilizing the proposed method, the distribution of stresses inside a sheet pile can be determined with comparably small computational effort. All through the paper, it is assumed that the sheets are not glued or laminated, but interact through frictional contact only.

In previous works of the authors $[1,2]$, a suitable macroscopic material law for the computational simulation of a single thin-sheet pile was proposed in total formulation for two dimensional problems. In the present paper, an according incremental material law formulation is presented. Additionally, this material law is extended, such that more general problem setups can be considered. Instead of modelling a single pile as in $[1,2]$, several piles which interact through a frictional connection are considered. The frictional connection is realized by mutual overlapping of sheet stacks. In applications, such tight overlaps are frequently realized in order to connect different piles frictionally. A simple model setup consisting of two interfingering piles is given in Figure 1. Such a connection has a-priori no in-plane strength; only the application of a transversal compressive force leads to some stability of the connection.

A standard approach to the computational simulation of mechanical problems is the finite element method. Powerful finite element software packages such as ABAQUS [3] allow the numerical treatment of a large class of mechanical problem setups. However, in the present case of thin-sheet piles, conventional methods fail: industrial applications, e.g. the iron core of a large power transformer, include piles of dimension of several meters, while the thickness of a single sheet ranges below one millimeter. Thus, a straightforward finite element discretization of the geometry of a single pile leads to billions of elements. Additionally, contact conditions have to be imposed on thousands of interfaces. This leads to strongly coupled nonlinearities and extremely large systems of equations, for which a numerical treatment becomes almost or truly impossible.

The usage of a macroscopic material model for an equivalent homogenized computational domain, as suggested in this paper, is inspired by well-known techniques. In the literature, such approaches are referred to as homogenization of materials with periodic micro structure. An extensive overview on homogenization techniques is given in the monograph by Nemat-Nasser and Hori [4]. Within this monograph, the solid with periodically distributed geometrical or material inhomogeneities is replaced by a homogenized solid, the periodical elasticity tensor is replaced by a matching constant tensor. For the material at hand and considering a pile of sheets, the inhomogeneities at the micro-level matches the contact conditions on the sheet interfaces and the homogenized solid at the macro-level corresponds to the pile of sheets. The contact conditions can be interpreted as local highly nonlinear springs, which are embedded into a global material law in the homogenized case. Inspired by these techniques at hand, the overall response of the system is modeled in an averaged sense. As presented in [4], for general homogenization techniques a mapping back to the microstructure is possible. In the present paper this mapping to the single sheet level 
is not treated, since only the global response of the system is of interest.

To date, many contributions concerning homogenization techniques for composite solids exist. Approaches for layered materials can be found e.g. in $[5,6]$. However, the different layers are always laminated or glued, while in the problem at hand sheets interact through frictional contact only. Existing models on delamination and crack analysis, such as $[7,8]$, cannot be applied directly: in these models, single small micro-cracks are considered, while the current problem involves thousands of macro-scale contact interfaces. Homogenization methods have also been developed for the numerical simulation of masonry structures, see e.g. Sacco [9], in which elastic bricks are connected by weak layers of mortar, in which cracks may develop.

The approach chosen in this paper is related to another technique developed in connection with masonry structures: the so-called no-tension material, which is introduced in the work of Heyman [10]. Theory on no-tension materials is provided in $[11,12]$. Different variants of the no-tension material were applied in the simulation of brickwork or masonry $[13,14,15]$. It is a commonly accepted fact that the no-tension model can give useful results concerning the overall structural behavior and arising stress distributions in old masonry structures. In [16], admissible static loads for a panel of no-tension material are discussed. In contrast to no-tension materials, in the present case the strength of the material is anisotropic due to the layered structure of the pile. While the former materials cannot withstand tension in any direction, the material developed in the present paper does not allow for tensile forces orthogonal to the sheet plane, while the shear strain is limited by friction.

The current approach is mechanically motivated by and based on well-known contact conditions and Coulomb's friction law, which can e.g. be found in the book by Wriggers [17]. The conditions for frictional contact are posed not only on sheet interfaces, but everywhere in the body. This task was accomplished for the case of a single pile in two dimensions in preceding works [1, 2]. Frictional connections have been treated by the authors [18] in two dimensions, the spatial case was shortly addressed in [19]. This means that no tension orthogonal to the sheet plane may arise, and that the shear stresses have to suffice Coulomb's friction law. This leads to a block behaving like a pile of very thin sheets with no bending stiffness. This material model proved sufficiently accurate for the desired applications. In the present paper, this approach shall be extended to the treatment of regions where several piles interact through mutual overlapping. In such regions of interfingering, the in-plane stress is bounded in terms of the compressive force and various parameters describing the frictional connection within the so called overlapping region. The obtained material model is of similar form as models in elasto-plasticity, using the well-known duality between contact and plasticity, see e.g. [20]. Thus, an according numerical treatment is proposed.

In applications, homogenized blocks obeying the new material law can be discretized much coarser than the complex geometry of the interconnected sheet piles. Larger elements can be used, while the accuracy of the solution and the overall response can be kept at a satisfactory level. The number of degrees of 
freedom is reduced dramatically, which also leads to better numerical stability of the nonlinear system, and faster convergence than for the original system.

\section{Problem description}

A macroscopic material law for the simulation of thin-sheet piles is developed. The model is phenomenologically motivated; contact conditions, which bound the contact forces on sheet interfaces for the original model, are posed in the whole homogenized domain in an average sense. Compressive and shear stresses are bounded to obey the contact conditions locally everywhere in the equivalent homogenized body. The material law is characterized by according unilateral constraint functions on the stress: in direction normal to the sheet plane compressive forces are allowed only, and in the tangential sheet plane the absolute value of the shear stress must obey Coulomb's law of friction. These constraints are described shortly in Section 3.2 and Section 3.3, respectively.

In applications, a connection of piles is achieved by means of a tight interlock, where sheets from both piles overlap mutually. For an exemplary problem setup, see Figure 1. Such a connection can a-priori withstand no in-plane tension and the piles may be moved relatively. By applying a compressive force orthogonal to the overlaps of this connection, some in-plane strength of the connection is gained by the friction acting between the overlapping interfaces of the piles. Relative movements of the two piles due to small in-plane forces are then blocked. In simulations, the macroscopic material model mentioned above cannot be applied directly in such connecting regions. Thus, the above model is extended, such that it represents the behavior of sheet piles connected by regions of mutual overlapping. For the homogenized block, all relative motions of sheets are modeled by a continuous deformation. In Figure 2, such a continuous deformation representing a shift of the piles of sheets is visualized. The according constraints are described in Section 3.4.

\subsection{Model setup and necessary assumptions}

In the sequel, a model problem consisting of two different piles connected in one overlapping region shall be considered. For a sketch of the model setup, see Figure 1. A macroscopic material model shall be developed, which allows to treat the piles as a single equivalent homogenized block, which is divided in three sub-regions: two sub-regions corresponding to the piles, and a third matching the region of mutual overlapping between the two blocks. Throughout the remainder of this paper, the left and right parts shall be referred to as "single pile regions", while the connecting part is called "overlapping region". For the single pile regions, a macroscopic material model imitating frictional contact of thin sheets is proposed. In the overlapping region, an extension of this material model is discussed.

For the geometric description of the overlaps, the following convention is introduced: the height of the whole overlapping region shall be addressed by $h_{o l}$, while $N_{o l}$ is the total number of in-plane overlap interfaces. For large numbers of 
overlaps, then $h_{o l} / N_{o l}$ is approximately the thickness of one overlapping stack. The "overlap length" $l_{o l}$ shall denote the axial length of the interfaces, which can be defined as the distance between the two single pile regions. For the twopile model problem, additionally the "overlap width" $w_{o l}$ can be defined as the third measure defining the overlapping region, and is equal to the depth of the two piles.

A local coordinate system $\left(\mathbf{e}_{x}^{*}, \mathbf{e}_{y}^{*}, \mathbf{e}_{z}^{*}\right)$ is attached to the homogenized block, which allows to identify the directions of anisotropic behavior of the layered, mutually overlapping sheets. The coordinate vector $\mathbf{e}_{z}^{*}$ shall be orthogonal to the sheet plane. According to this choice, the sheet plane shall sometimes be considered as horizontal, while the direction orthogonal to this plane is referred to as vertical. The $\mathbf{e}_{x}^{*}$ and $\mathbf{e}_{y}^{*}$ coordinates are aligned with the overlapping region in such a way that $\mathbf{e}_{x}^{*}$ points from pile 1 to pile 2 , while $\mathbf{e}_{y}^{*}$ is chosen such that the triplet $\left(\mathbf{e}_{x}^{*}, \mathbf{e}_{y}^{*}, \mathbf{e}_{z}^{*}\right)$ forms a Cartesian coordinate system. Then, $\mathbf{e}_{x}^{*}$ is aligned with the overlap length $l_{o l}$, while $\mathbf{e}_{y}^{*}$ is aligned with the overlap width $w_{o l}$. The macroscopic material law used in single pile regions is described by the material friction parameter $\mu$ and the transverse direction $\mathbf{e}_{z}^{*}$ only. For the description of the local material behavior in overlapping regions, the following set of parameters is sufficient

- the axial length of the overlapping region $l_{o l}$,

- the number of overlap interfaces per unit height $\rho_{o l}$,

- the material friction parameter $\mu$,

- the local coordinate system $\left(\mathbf{e}_{x}^{*}, \mathbf{e}_{y}^{*}, \mathbf{e}_{z}^{*}\right)$.

The parameter $\rho_{o l}=N_{o l} / h_{o l}$ depends on the number of overlap interfaces in total, $N_{o l}$, and the height of the overlapping region $h_{o l}$.

Throughout the sequel, the following two assumptions regarding the geometrical setup and the external and internal forces acting in the equivalent homogenized body are made:

Assumption 1. All deformations and strains are small, such that the theory of linearized elasticity can be applied.

Assumption 2. The single elastic sheets are thin, such that their bending stiffness can be neglected and homogenization of contact conditions is admissible.

Assumption 3. No local external forces act, which lead to a relative displacement of single sheets, i.e. all external forces are distributed over many sheets or a long area.

\section{A nonlinear macroscopic material law}

In the present paper, all vector and tensor fields are denoted as boldface letters, second-order tensors are moreover underlined. The displacement vector 
is denoted by $\mathbf{u}$, while $\underline{\varepsilon}=\underline{\varepsilon}(\mathbf{u})$ is the linearized strain tensor defined by

$$
\underline{\varepsilon}(\mathbf{u})=\frac{1}{2}\left(\nabla \mathbf{u}+\nabla \mathbf{u}^{T}\right) .
$$

In the current work, it is assumed that the deformation of each single sheet is small, such that it stays in the linear elastic range. Then, for each sheet, the stress tensor $\underline{\boldsymbol{\sigma}}$ depends linearly on the strain $\underline{\boldsymbol{\varepsilon}}$,

$$
\underline{\sigma}=\underline{\underline{D}} \underline{\varepsilon}(\mathbf{u}) \text {. }
$$

The fourth order tensor $\mathbf{D}$ is the elasticity tensor, which is characterized by

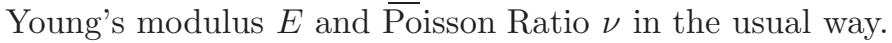

All considerations concerning the macroscopic material have to be done in the local coordinate system $\left(\mathbf{e}_{x}^{*}, \mathbf{e}_{y}^{*}, \mathbf{e}_{z}^{*}\right)$. Let therefore, for $\alpha, \beta \in\{x, y, z\}$

$$
\begin{aligned}
\sigma_{\alpha, \beta}^{*} & =\left(\mathbf{e}_{\alpha}^{*}\right)^{T} \underline{\boldsymbol{\sigma}} \mathbf{e}_{\beta}^{*}, \\
\varepsilon_{\alpha, \beta}^{*} & =\left(\mathbf{e}_{\alpha}^{*}\right)^{T} \underline{\boldsymbol{\varepsilon}} \mathbf{e}_{\beta}^{*}
\end{aligned}
$$

be stress and strain components with respect to the local coordinate system. To avoid unnecessary complications, however, throughout the remainder of this paper we assume that the local coordinate system is identical to the global one, such that all asterisks can be omitted. Otherwise, the transformed quantities given in equations (3) and (4) have to be used.

\subsection{General framework for the macroscopic material law}

The macroscopic material law is nonlinear, and modeled similar to elastoplastic material laws. Different constraint functions, which are treated similar to yield functions in plasticity, describe the stress-strain relationship in the homogenized block. Typically, such a constraint function will be given in the form

$$
f(\underline{\boldsymbol{\sigma}}) \leq 0 .
$$

For a detailed introduction into the theory of plasticity, the reader is referred to the monographs of Ziegler [21], Simo and Hughes [22] or Dill [23]. The stressstrain relationship is no longer linear. In the current approach, the strain tensor $\underline{\varepsilon}$ is split into an elastic and an inelastic part,

$$
\underline{\varepsilon}=\underline{\varepsilon}^{e}+\underline{\varepsilon}^{i} .
$$

Thus, the inelastic part of strain $\underline{\varepsilon}^{i}$ is introduced, which corresponds to the averaged relative movements of the sheets, as will be motivated in more detail later. A similar approach was used by Angelillo [12] for the description of masonry structures. In this work, the inelastic quantity is referred to as "additive free deformation". The stress depends then on the elastic part of strain $\underline{\varepsilon}^{e}=\underline{\varepsilon}-\underline{\varepsilon}^{i}$,

$$
\underline{\mathbf{\sigma}}=\underline{\underline{\mathbf{D}}} \underline{\underline{\varepsilon}}^{e}=\underline{\underline{\mathbf{D}}}\left(\underline{\varepsilon}-\underline{\varepsilon}^{i}\right) .
$$


Using the virtual work of external forces $\delta W_{e x t}$, the equations of virtual work for the macroscopic material read

$$
\int_{V} \underline{\underline{\mathbf{D}}}\left(\underline{\varepsilon}-\underline{\varepsilon}^{i}\right): \delta \underline{\varepsilon} d V=\delta W_{e x t} .
$$

As in plasticity, the inelastic strain $\underline{\varepsilon}^{i}$ may change in time (i.e. $\underline{\dot{\varepsilon}}^{i} \neq 0$ ) only as long as the condition $f(\underline{\boldsymbol{\sigma}})=0$ is satisfied. In plasticity, the set of stresses for which $f(\underline{\boldsymbol{\sigma}})=0$ holds is addressed as yield surface. If, on the other hand, $f(\underline{\boldsymbol{\sigma}})<0$, the inelastic strain rate must vanish, $\underline{\dot{\varepsilon}}^{i}=0$. This relation between yield condition and inelastic strain rate can be rewritten equivalently as

$$
f(\underline{\boldsymbol{\sigma}}) \underline{\dot{\varepsilon}}^{i}=0 .
$$

As mentioned above, the inelastic part of strain is closely connected to the relative movement of adjacent sheets. Thus, condition (9) ensures that a relative movement of sheets may occur only if the respective contact condition $f(\underline{\boldsymbol{\sigma}}) \leq 0$ is satisfied with equality on a local stress level. In case of $f(\underline{\boldsymbol{\sigma}})=0$, an update rule for the inelastic part of strain has to be given, which determines the inelastic strain rate $\underline{\dot{\varepsilon}}^{i}$ depending on $\underline{\boldsymbol{\sigma}}$. This behavior is mathematically represented by the Kuhn-Tucker type conditions

$$
\begin{aligned}
f(\underline{\boldsymbol{\sigma}}) & \leq 0, \\
\underline{\dot{\varepsilon}}^{i} & =\lambda \underline{\mathbf{g}} \quad \text { with } \lambda \geq 0, \\
f(\underline{\boldsymbol{\sigma}}) \underline{\dot{\varepsilon}}^{i} & =\mathbf{0} .
\end{aligned}
$$

Here, $\mathbf{g}$ is the update direction for the inelastic part of strain, which has to be specified. In plasticity, the strain rate is chosen in direction orthogonal to the yield surface, as proposed e.g. by Dill [23, Chapter 4]. This results in setting the inelastic part of strain increment as a multiple of $\mathbf{g}=\partial f / \partial \underline{\mathbf{\sigma}}$. Such a behavior is referred to as associative plasticity. For the homogenized material developed in the sequel, both associative and non-associative update rules are proposed. In Section 3.2 and Section 3.3, update rules proposed in [1, 2] for the normal and tangential contact are provided. In Section 3.4, an appropriate unilateral constraint function for the description of the in-plane strength of a connection via an overlapping region is given. A matching update rule is proposed and analyzed. The inelastic part of strain tensor will be split additively into components accounting for the normal contact, the friction conditions, and the additional conditions in the overlapping regions, which are denoted by $\underline{\varepsilon}_{N}^{i}$, $\underline{\boldsymbol{\varepsilon}}_{T}^{i}$ and $\underline{\varepsilon}_{o l}^{i}$, respectively,

$$
\underline{\varepsilon}^{i}=\underline{\varepsilon}_{N}^{i}+\underline{\varepsilon}_{T}^{i}+\underline{\varepsilon}_{o l}^{i} .
$$

The impact of the different contact phenomena is split on different strain components, which makes it easy assignable. As will be seen later, only certain components of the three latter tensors are non-zero, in total representing the 
inelastic part of strain, namely

$$
\underline{\varepsilon}_{N}^{i}:=\left[\begin{array}{ccc}
0 & 0 & 0 \\
0 & 0 & 0 \\
0 & 0 & \varepsilon_{z z}^{i}
\end{array}\right], \quad \underline{\varepsilon}_{T}^{i}:=\left[\begin{array}{ccc}
0 & 0 & \varepsilon_{x z}^{i} \\
0 & 0 & \varepsilon_{y z}^{i} \\
\varepsilon_{x z}^{i} & \varepsilon_{y z}^{i} & 0
\end{array}\right], \quad \underline{\varepsilon}_{o l}^{i}:=\left[\begin{array}{ccc}
\varepsilon_{x x}^{i} & \varepsilon_{x y}^{i} & 0 \\
\varepsilon_{x y}^{i} & \varepsilon_{y y}^{i} & 0 \\
0 & 0 & 0
\end{array}\right] .
$$

Thus, the splitting in Eq. (13) is unique. In the same manner, stress and strain can be split additively, setting

$$
\underline{\varepsilon}=\underline{\varepsilon}_{N}+\underline{\varepsilon}_{T}+\underline{\varepsilon}_{o l}, \quad \underline{\sigma}=\underline{\sigma}_{N}+\underline{\sigma}_{T}+\underline{\sigma}_{o l} .
$$

These matrices shall be used as additive quantities in a mathematical sense only, they do not represent physical stress or strain states.

\subsection{Normal contact}

Recall that the sheets are not glued but interact through frictional contact only. This implies that no tensile surface stress orthogonal to the sheet plane can appear on sheet interfaces. For the macroscopic material model, this condition is posed everywhere in the homogenized domain, assuming that the sheet thickness is much smaller than the total height of the pile of sheets. The latter condition implies that the material cannot take any tensile stresses in direction normal to the sheet plane $\mathbf{e}_{z}$. This leads to the inequality constraint

$$
f_{N}(\underline{\boldsymbol{\sigma}})=\sigma_{z z} \leq 0 \text {. }
$$

In case of $f_{N}(\underline{\boldsymbol{\sigma}})=0$, the inelastic part of strain rate is chosen as a multiple of $\underline{\mathrm{g}}_{N}:=\partial f_{N} / \partial \underline{\boldsymbol{\sigma}}$, which results in a transverse strain component rate $\underline{\dot{\varepsilon}}_{N}^{i}$,

$$
\underline{\dot{\varepsilon}}_{N}^{i}=\lambda_{N} \underline{\mathbf{g}}_{N}=\lambda_{N}\left[\begin{array}{ccc}
0 & 0 & 0 \\
0 & 0 & 0 \\
0 & 0 & 1
\end{array}\right]
$$

with non-negative Lagrangian multiplier $\lambda_{N} \geq 0$. In case of an opening of the sheets (positive gap), the unknown multiplier $\lambda_{N}$ needs to be determined from the inequality constraint of the normal force Eq. (16). Since in this case the inelastic strain rate $\underline{\dot{\varepsilon}}_{N}^{i}=\lambda_{N} \underline{\mathbf{g}}_{N} \neq 0$, according to Eq. (9) the inequality constraint (16) has to be satisfied with equality, $f_{N}(\underline{\boldsymbol{\sigma}})=0$. Since the stress depends on the inelastic strain, one can derive a corresponding equation for $\lambda_{N}$, which is not provided here. .

\subsection{Frictional contact}

In the general case of contacting bodies, Coulomb's law of friction has to hold on each sheet interface. The relation of tangential contact force (=friction force) vector $\mathbf{F}_{T}$, material friction parameter $\mu$, and normal contact force $F_{N}$ is given as

$$
\left\|\mathbf{F}_{T}\right\| \leq \mu\left|F_{N}\right|,
$$


where $\|\cdot\|$ denotes the Euclidean norm of a vector. In the macroscopic material, Coulomb's law of friction is applied in a local form. The tangential contact force vector $\mathbf{F}_{T}$ is related to the shear stress vector $\boldsymbol{\sigma}_{T}$, which is defined along with the shear strain vector $\boldsymbol{\varepsilon}_{T}$ by

$$
\boldsymbol{\sigma}_{T}:=\left[\begin{array}{c}
\sigma_{x z} \\
\sigma_{y z} \\
0
\end{array}\right], \quad \boldsymbol{\varepsilon}_{T}:=\left[\begin{array}{c}
\varepsilon_{x z} \\
\varepsilon_{y z} \\
0
\end{array}\right],
$$

Note that these vectors correspond to the tensor components $\underline{\boldsymbol{\varepsilon}}_{T}$ and $\underline{\boldsymbol{\sigma}}_{T}$ defined by the additive splitting (15). On the basis of Coulomb's friction law (18) assigned to the stress level, the absolute value of the stress vector $\boldsymbol{\sigma}_{T}$ is bounded by the friction coefficient $\mu$ times the absolute value of the compressive stress $\sigma_{z z}$,

$$
f_{T}(\boldsymbol{\sigma}):=\left\|\boldsymbol{\sigma}_{T}\right\|-\mu\left|\sigma_{z z}\right| \leq 0 .
$$

This condition is similar to the Mohr-Coulomb yield condition (see e.g. the monograph of Mang and Hofstetter [24], which is used for brittle materials or rubble piles. In the present case, the anisotropic nature of the sheet pile with respect to the vertical $\mathbf{e}_{z}$-direction is incorporated.

Choosing the inelastic strain rate $\underline{\dot{\varepsilon}}_{T}^{i}$ as a multiple of $\partial f_{T} / \partial \underline{\boldsymbol{\sigma}}$ leads to a non-vanishing rate of the inelastic shear strain component $\underline{\dot{\varepsilon}}_{T}^{i}$ as well as of the inelastic transverse strain $\underline{\dot{\varepsilon}}_{N}^{i}$, since the constraint function $f_{T}(\underline{\boldsymbol{\sigma}})$ depends not only on $\boldsymbol{\sigma}_{T}$, but also on the normal stress component $\sigma_{z z}$. Instead, an approach motivated from contact mechanics is used, where relative sliding of the contact interfaces occurs in direction of the tangential stress vector $\boldsymbol{\sigma}_{T}$. In the framework of constraint functions and yield rules, a different constraint function $f_{T}^{*}$ with fixed compressive stress $\sigma_{z z}^{0}$ is used,

$$
f_{T}^{*}(\boldsymbol{\sigma}):=\left\|\boldsymbol{\sigma}_{T}\right\|-\mu\left|\sigma_{z z}^{0}\right| .
$$

The inelastic strain rate $\underline{\dot{\varepsilon}}_{T}^{i}$ is proposed to be proportional to $\underline{\mathbf{g}}_{T}:=\partial f_{T}^{*} / \partial \underline{\mathbf{\sigma}}$, which leads to

$$
\underline{\dot{\varepsilon}}_{T}^{i}:=\lambda_{T} \underline{\mathbf{g}}_{T}=\frac{\lambda_{T}}{\left\|\boldsymbol{\sigma}_{T}\right\|}\left[\begin{array}{ccc}
0 & 0 & \sigma_{x z} \\
0 & 0 & \sigma_{y z} \\
\sigma_{x z} & \sigma_{y z} & 0
\end{array}\right] .
$$

with $\lambda_{T} \geq 0$. The inelastic strain component $\underline{\varepsilon}_{T}^{i}$ corresponds to the relative sliding movement in the tangential plane of the contact interfaces, which is the $\mathbf{e}_{x} \mathbf{e}_{y}$-plane here. The dimensionless tensor component $\underline{\mathbf{g}}_{T}$ is related to the local direction of sliding $\boldsymbol{\sigma}_{T}$, while the scalar $\lambda_{T}$ is related to the absolute value of the relative displacement.

\subsection{Frictional connections}

A third constraint function, which accounts for the overlaps of a frictional connection of two piles, is derived subsequently. This overlapping region is not only anisotropic with respect to the $\mathbf{e}_{z}^{*}$-direction, but also with respect to 
the local in-plane coordinates $\mathbf{e}_{x}^{*}$ and $\mathbf{e}_{y}^{*}$. Thus, recall that all considerations have to be done using the local coordinate system $\left(\mathbf{e}_{x}^{*}, \mathbf{e}_{y}^{*}, \mathbf{e}_{z}^{*}\right)$, where tensor components are computed according to Eqns. (3) and (4). In order not to complicate notation, all asterisks are omitted in the following.

Let $F_{N}$ be the total compressive force in the overlapping region. In Figure 3(a), a connection of two piles via one single horizontal interface is depicted. For this case, the Euclidean norm of the in-plane force vector $\mathbf{F}_{o l}=$ $\left[\begin{array}{lll}F_{o l, x} & F_{o l, y} & 0\end{array}\right]^{T}$ acting on a surface orthogonal to $\mathbf{e}_{x}$ is limited by the material friction coefficient $\mu$ times $\left|F_{N}\right|,\left\|\mathbf{F}_{o l}\right\| \leq \mu\left|F_{N}\right|$. In the case under consideration, there are $N_{o l}$ interfaces of stacks from left and right pile. This leads to $N_{o l}$ sliding surfaces, and the necessary in-plane force is multiplied by a factor $N_{o l}$, as is depicted in Figure 3(b). Thus the necessary condition for sliding of the two piles reads

$$
\left\|\mathbf{F}_{o l}\right\| \leq \mu N_{o l}\left|F_{N}\right| .
$$

In order to transfer condition (23) to the stress level, a hexahedral of infinitesimal height $d z$ and width $d y$ is considered. The hexahedral shall be of macroscopic length $l_{o l}$ in direction $\mathbf{e}_{x}$, such that it extends over the whole overlap length. In a finite element implementation, this volume may be resolved by several finite elements along the macroscopic length $l_{o l}$ in $x$-direction. For a sketch of the hexahedral including the single sheets, see Figure 4(a). The homogenized equivalent is displayed in Figure 4(b). Over the height of this hexahedral, $N_{o l}=\rho_{o l} d z$ overlap interfaces are expected on average. The total compressive force $F_{N}$ from Eq. (23) is related to the compressive transverse stress $\bar{\sigma}_{z z}$ averaged over the overlap length,

$$
F_{N}=l_{o l} d y \bar{\sigma}_{z z} \quad \text { with } \quad \bar{\sigma}_{z z}(y, z)=\int_{0}^{l_{o l}} \sigma_{z z}(x, y, z) d x .
$$

The in-plane force vector $\mathbf{F}_{o l}$ corresponds to the in-plane stress vector $\boldsymbol{\sigma}_{o l}$, as sketched in Figure 4

$$
\mathbf{F}_{o l}=d y d z \boldsymbol{\sigma}_{o l} \quad \text { with } \boldsymbol{\sigma}_{o l}:=\left[\begin{array}{c}
\sigma_{x x} \\
\sigma_{x y} \\
0
\end{array}\right] .
$$

Note that the in-plane stress vector $\boldsymbol{\sigma}_{o l}$ corresponds to the stress component $\underline{\boldsymbol{\sigma}}_{o l}$ defined by the additive splitting (15). The necessity of the averaging procedure in Eq. (24) can be motivated as follows: a relative motion of the piles in direction $\mathbf{e}_{x}$ takes place only if the in-plane force $\mathbf{F}_{o l}$ reaches the critical value with respect to the total compressive force $F_{N}$ in (24). Then, the interfaces slide along the whole overlap length. In case the overlapping connection is discretized by several finite elements in $\mathbf{e}_{x}$ direction, the averaging (24) is done over all these elements.

Inserting the representations from Eq. (24) and Eq. (25) into the constraint on the in-plane force from Eq. (23) and using that the number of overlap interfaces is $\rho_{o l} d z$ for the infinitesimal cube leads to the bound

$$
d y d z\left\|\boldsymbol{\sigma}_{o l}\right\| \leq \mu \rho_{o l} l_{o l} d y d z\left|\bar{\sigma}_{z z}\right| .
$$


Expressing the corresponding bound by an according constraint function $f_{o l}$ leads to

$$
f_{o l}(\underline{\boldsymbol{\sigma}}):=\left\|\boldsymbol{\sigma}_{o l}\right\|-\mu \rho_{o l} l_{o l}\left|\bar{\sigma}_{z z}\right| \leq 0 .
$$

In the following, an update law for the inelastic part of strain rate $\dot{\boldsymbol{\varepsilon}}_{o l}^{i}$ concerning the overlap constraint function $f_{o l}$ shall be developed. Therefore, the non-trivial case $f_{o l}(\boldsymbol{\sigma})=0$ is assumed, since otherwise the inelastic strain component $\underline{\varepsilon}_{o l}^{i}$ remains constant by setting $\underline{\dot{\varepsilon}}_{o l}^{i}=0$, as implied by the Kuhn-Tucker type conditions (9). It will turn out that the definition of the additive inelastic part of strain component $\underline{\varepsilon}_{o l}^{i}$ in Eq. (14) was an appropriate choice for the constraint function $f_{o l}$.

As in the case of tangential contact, a constraint function $f_{o l}^{*}(\underline{\boldsymbol{\sigma}})$ is introduced, where the average compressive stress $\bar{\sigma}_{z z}$ is fixed at a constant value $\bar{\sigma}_{z z}^{0}$,

$$
f_{o l}^{*}(\underline{\boldsymbol{\sigma}}):=\left\|\boldsymbol{\sigma}_{o l}\right\|-\mu \rho_{o l} l_{o l}\left|\bar{\sigma}_{z z}^{0}\right| .
$$

Again, the inelastic strain rate is chosen as a multiple of an update direction $\underline{\mathrm{g}}_{o l}$,

$$
\underline{\dot{\varepsilon}}_{o l}^{i}=\lambda_{o l} \underline{\mathrm{g}}_{o l} .
$$

However, in contrast to the cases of normal and tangential contact, no associative flow rule is applicative in this case. The choice of $\underline{\mathbf{g}}_{o l}$ shall be motivated in the following phenomenological considerations in the infinitesimal volume $\left(l_{o l}, d y, d z\right)$ from Figure $4(\mathrm{~b})$. In case of sliding overlaps $\left(f_{o l}(\underline{\boldsymbol{\sigma}})=0\right)$, the stress vector $\boldsymbol{\sigma}_{o l}$ acting on the $\mathbf{e}_{y} \mathbf{e}_{z}$-plane leads to a relative displacement of the two piles in this direction. In Figure 5, this relative displacement of the two piles is depicted, where the infinitesimal hexahedral from Figure 4(b) is depicted in top view. Using the relative displacement rate $\dot{\mathbf{u}}^{r}$ of the two piles, this fact is described by the relation

$$
\dot{\mathbf{u}}^{r}=\frac{\lambda_{o l}}{\left\|\boldsymbol{\sigma}_{o l}\right\|} \boldsymbol{\sigma}_{o l} .
$$

The scalar $\lambda_{o l}$ is the absolute value of the relative displacement, and acts as a Lagrangian multiplier.

In the homogenized setup, a corresponding continuous "inelastic displacement" $\mathbf{u}^{i}$ has to be used. In Figure 6, the homogenized equivalent of Figure 5 is shown. In the infinitesimal region, the continuous inelastic displacement is obtained from a linear interpolation of the relative displacement increment $\dot{\mathbf{u}}^{r}$ with respect to the $x$-direction along the length $l_{o l}$. This interpolated inelastic displacement is given by

$$
\dot{\mathbf{u}}^{i}=x \frac{\lambda_{o l}}{\left\|\boldsymbol{\sigma}_{o l}\right\|} \boldsymbol{\sigma}_{o l} .
$$

Using the displacement gradient increment $\nabla \dot{\mathbf{u}}^{i}$, one obtains a local formulation for the direction of the inelastic rate

$$
\nabla \dot{\mathbf{u}}^{i}=\frac{\lambda_{o l}}{\left\|\boldsymbol{\sigma}_{o l}\right\|}\left[\begin{array}{ccc}
\sigma_{x x} & 0 & 0 \\
\sigma_{x y} & 0 & 0 \\
0 & 0 & 0
\end{array}\right] .
$$


The corresponding (linearized) inelastic part of strain tensor reads then

$$
\underline{\dot{\varepsilon}}_{o l}^{i}=\frac{1}{2}\left(\nabla \dot{\mathbf{u}}^{i}+\left(\nabla \dot{\mathbf{u}}^{i}\right)^{T}\right)=\frac{\lambda_{o l}}{\left\|\boldsymbol{\sigma}_{o l}\right\|}\left[\begin{array}{ccc}
\sigma_{x x} & \frac{1}{2} \sigma_{x y} & 0 \\
\frac{1}{2} \sigma_{x y} & 0 & 0 \\
0 & 0 & 0
\end{array}\right]=\lambda_{o l} \underline{\mathbf{g}}_{o l},
$$

with the update direction $\underline{\mathrm{g}}_{o l}$ defined by

$$
\underline{\mathrm{g}}_{o l}:=\frac{1}{\left\|\boldsymbol{\sigma}_{o l}\right\|}\left[\begin{array}{ccc}
\sigma_{x x} & \frac{1}{2} \sigma_{x y} & 0 \\
\frac{1}{2} \sigma_{x y} & 0 & 0 \\
0 & 0 & 0
\end{array}\right] .
$$

According to the above definition of $\underline{\mathbf{g}}_{o l}$, the inelastic strain rate $\underline{\boldsymbol{\varepsilon}}_{o l}^{i}$ is set according to Eq. (29).

Note that the described constraint functions (16), (20) and (27) can also be considered in an multi-surface plasticity framework [25].

\section{Computational treatment of the macroscopic material law}

In the sequel, the algorithmic treatment of the nonlinear macroscopic material law in a computational code shall be discussed. To this end, it is assumed that the homogenized block is discretized into finite elements resolving the borders of the overlapping region, but not the single sheet level. An iterative procedure for the computation of the inelastic quantities is proposed. The three constraint functions $f_{N}, f_{T}$ and $f_{o l}$ are treated consecutively, as inspired by contact mechanics: first, the inelastic part of strain $\underline{\varepsilon}_{N}^{i}$ is computed, which determines the contact pressure $\sigma_{z z}$. For a fixed value of $\underline{\varepsilon}_{N}^{i}$ and thereby a fixed contact pressure, values for $\underline{\varepsilon}_{T}^{i}$ corresponding to sliding motions and $\underline{\varepsilon}_{o l}^{i}$ corresponding to relative displacements of piles are obtained. The authors point out that the order of the sequence of the updates is important. However, for fixed contact pressure, the two later updates are independent, and can be done in any order, since different components of the inelastic part of strain tensor are concerned. In each element, the inelastic strains are approximated in the integration points of an appropriate Gaussian quadrature rule. The conditions of the nonlinear macroscopic material law given in Eqns. (16), (20) and (27) are imposed in these integration points. The following notation is specified for the iterative step $k$ :

- values associated to iterative step $k$ are labeled by an upper index $k$, such as the displacement vector $\mathbf{u}^{k}$, the strain tensor $\underline{\varepsilon}^{k}=\underline{\varepsilon}\left(\mathbf{u}^{k}\right)$, the inelastic strain $\underline{\varepsilon}^{i, k}$, and the stress tensor $\underline{\mathbf{\sigma}}^{k}=\underline{\underline{\mathbf{D}}}\left(\underline{\varepsilon}^{k}-\underline{\varepsilon}^{i, k}\right)$,

- in step $k$, values for $\mathbf{u}^{k-1}$ and $\underline{\varepsilon}^{i, k-1}$, which were approved in the previous step, are given,

- inelastic strains from step $k-1$ and $k$ are linked by the inelastic strain update $d \underline{\varepsilon}^{i, k}$ via

$$
\underline{\boldsymbol{\varepsilon}}^{i, k}=\underline{\boldsymbol{\varepsilon}}^{i, k-1}+d \underline{\underline{\varepsilon}}^{i, k} .
$$


This strain update is split uniquely into three independent quantities, $d \underline{\varepsilon}^{i, k}=d \underline{\varepsilon}_{N}^{i, k}+d \underline{\varepsilon}_{T}^{i, k}+d \underline{\varepsilon}_{o l}^{i, k}$ as implied by Eq. (14).

It is proposed to employ a return mapping type iteration procedure, which is sketched briefly in the following:

Algorithm 1. Given an initial inelastic strain $\underline{\underline{\varepsilon}}^{i, 0}$, starting from $k=1$ iterate:

1. compute $\mathbf{u}^{k}$ as a solution to the equation of virtual work (8) using the given inelastic strain $\underline{\underline{\varepsilon}}^{i, k-1}$,

2. if the stress tensor $\underline{\mathbf{\sigma}}^{k}:=\underline{\mathbf{D}}\left(\underline{\varepsilon}\left(\mathbf{u}^{k}\right)-\underline{\varepsilon}^{i, k-1}\right)$ satisfies the complementarity condition Eq. (9) for $f_{N}, f_{T}$ and $f_{o l}$ in all integration points with given error tolerance, return, else,

3. compute the inelastic strain update $d \underline{\varepsilon}_{N}^{i, k}$ according to Section 4.1,

4. compute the inelastic strain update $d \underline{\varepsilon}_{T}^{i, k}$ according to Section 4.2,

5. compute the inelastic strain update $d \underline{\varepsilon}_{o l}^{i, k}$ according to Section 4.3,

6. goto step 1.

In the following, update laws for the inelastic strain updates $d \underline{\varepsilon}_{N}^{i, k}, d \underline{\varepsilon}_{T}^{i, k}$ and $d \underline{\varepsilon}_{o l}^{i, k}$ shall be developed. The update laws are based on a return mapping algorithm, which is frequently used in elasto-plastic computations. The updates are done consecutively for the three different constraint functions in an appropriately ordered sequence. To this end, it is assumed that in iterative step $k$, an inelastic strain $\underline{\varepsilon}^{i, k-1}$ from the previous step is given, and that $\mathbf{u}^{k}$ has been computed according to Step 1 of Algorithm 1.

\subsection{Update rule for $d \underline{\varepsilon}_{N}^{i, k}$}

In Step 3 of Algorithm 1, it is required to find an update $d \underline{\varepsilon}_{N}^{i, k}$. Defining the intermediate stress quantity $\underline{\mathbf{\sigma}}^{0, k}$ and the update direction $\underline{\mathbf{g}}_{N}^{k}$ corresponding to Eq. (17)

$$
\underline{\boldsymbol{\sigma}}^{0, k}:=\underline{\underline{\mathbf{D}}}\left(\underline{\varepsilon}^{k}-\underline{\varepsilon}^{i, k-1}\right), \quad \underline{\mathbf{g}}_{N}^{k}:=\left[\begin{array}{ccc}
0 & 0 & 0 \\
0 & 0 & 0 \\
0 & 0 & 1
\end{array}\right],
$$

the update $d \underline{\varepsilon}_{N}^{i, k}$ is chosen in the following way

$$
d \underline{\varepsilon}_{N}^{i, k}:=\lambda_{N} \underline{\mathbf{g}}_{N}^{k} .
$$

The quantity $\lambda_{N}$ is still to be specified. In case of $f_{N}\left(\underline{\boldsymbol{\sigma}}^{0, k}\right) \leq 0$, when the contact pressure is non-positive, the inelastic strain update is set to zero, $d \underline{\varepsilon}_{N}^{i, k}=$ 0 . The case $f_{N}\left(\underline{\boldsymbol{\sigma}}^{0, k}\right)>0$ shall be considered in the following. A return mapping strategy is applied, where $\lambda_{N}$ is chosen such that the linearized constraint is satisfied with equality,

$$
\begin{aligned}
& f_{N}\left(\underline{\boldsymbol{\sigma}}^{0, k}\right)+\frac{\partial f_{N}}{\partial \underline{\boldsymbol{\sigma}}}\left(\underline{\boldsymbol{\sigma}}^{0, k}\right) \cdot\left(\underline{\boldsymbol{\sigma}}^{k}-\underline{\boldsymbol{\sigma}}^{0, k}\right)= \\
& f_{N}\left(\underline{\boldsymbol{\sigma}}^{0, k}\right)+\frac{\partial f_{N}}{\partial \underline{\boldsymbol{\sigma}}}\left(\underline{\boldsymbol{\sigma}}^{0, k}\right) \cdot\left(-\underline{\underline{\mathbf{D}}} d \underline{\boldsymbol{\varepsilon}}_{N}^{i, k}\right)=0 .
\end{aligned}
$$


Using that $\partial f_{N} / \partial \underline{\boldsymbol{\sigma}}=\underline{\mathbf{g}}_{N}^{k}$ and the update equation (37) leads to

$$
f_{N}\left(\underline{\boldsymbol{\sigma}}^{0, k}\right)-\lambda_{N} \underline{\mathbf{g}}_{N}^{k}:\left(\underline{\underline{\mathbf{D}}} \underline{\mathbf{g}}_{N}^{k}\right)=0 .
$$

This leads to the following choice for $\lambda_{N}$

$$
\lambda_{N}=\frac{f_{N}\left(\underline{\boldsymbol{\sigma}}^{0, k}\right)}{\underline{\mathbf{g}}_{N}^{k}:\left(\underline{\underline{\mathbf{D}}} \underline{\mathbf{g}}_{N}^{k}\right)}=\frac{f_{N}\left(\underline{\boldsymbol{\sigma}}^{0, k}\right)}{D_{z z z z}} .
$$

\subsection{Update rule for $d \underline{\varepsilon}_{T}^{i, k}$}

In Step 4 of Algorithm 1, an update $d \underline{\varepsilon}_{T}^{i, k}$ has to be computed. This is done similarly to the update computation for $d \underline{\varepsilon}_{N}^{i, k}$ described in Section 4.1. The intermediate stress quantity $\underline{\boldsymbol{\sigma}}^{0, k}$ is redefined such that it already contains the normal inelastic strain update,

$$
\underline{\boldsymbol{\sigma}}^{0, k}:=\underline{\underline{\mathbf{D}}}\left(\underline{\varepsilon}^{k}-\underline{\varepsilon}^{i, k-1}-d \underline{\boldsymbol{\varepsilon}}_{N}^{i, k}\right) .
$$

The tangential update direction corresponding to Eq. (22) reads

$$
\underline{\mathbf{g}}_{T}^{k}:=\frac{1}{\left\|\boldsymbol{\sigma}_{T}^{0, k}\right\|}\left[\begin{array}{ccc}
0 & 0 & \sigma_{x z}^{0, k} \\
0 & 0 & \sigma_{y z}^{0, k} \\
\sigma_{x z}^{0, k} & \sigma_{y z}^{0, k} & 0
\end{array}\right]=\frac{1}{\left\|\boldsymbol{\sigma}_{T}^{0, k}\right\|^{\boldsymbol{\sigma}_{T}^{0, k}} .}
$$

The update $d \underline{\boldsymbol{\varepsilon}}_{T}^{i, k}$ is chosen as a multiple of the update direction $\underline{\mathbf{g}}_{T}^{k}$,

$$
d \underline{\mathbf{s}}_{T}^{i, k}:=\lambda_{T} \underline{\mathbf{g}}_{T}^{k}
$$

The quantity $\lambda_{T}$ is set to zero in case the constraint inequality $f_{T}\left(\underline{\boldsymbol{\sigma}}^{0, k}\right) \leq 0$ is satisfied. Otherwise, $\lambda_{T}$ is chosen as a solution to the linearized constraint equation according to the return mapping scheme, which reads similar to the equation for $\lambda_{N}$ (38), but where for the derivative $\partial f_{T} / \partial \underline{\boldsymbol{\sigma}}$ instead of $f_{T}$ the constraint function $f_{T}^{*}$ with fixed compressive stress $\sigma_{z z}^{0}$ from Eq. (21) is used. Straightforward manipulations as already presented in Section 4.1 lead to

$$
f_{T}^{*}\left(\underline{\boldsymbol{\sigma}}^{0, k}\right)-\lambda_{T} \underline{\mathbf{g}}_{T}^{k}:\left(\underline{\underline{\mathbf{D}}} \underline{\mathbf{g}}_{T}^{k}\right)=0 .
$$

The parameter $\lambda_{T}$ is then chosen as

$$
\lambda_{T}=\frac{f_{T}\left(\underline{\boldsymbol{\sigma}}^{0, k}\right)}{\underline{\mathbf{g}}_{T}^{k}:\left(\underline{\underline{\mathbf{D}}} \underline{\mathbf{g}}_{T}^{k}\right)}=\left\|\boldsymbol{\sigma}_{T}^{0, k}\right\|^{2} \frac{f_{T}\left(\underline{\boldsymbol{\sigma}}^{0, k}\right)}{4 D_{x z x z}\left(\sigma_{x z}^{0, k}\right)^{2}+4 D_{y z y z}\left(\sigma_{y z}^{0, k}\right)^{2}} .
$$

This value is always well-defined in the case of $f_{T}\left(\underline{\boldsymbol{\sigma}}^{0, k}\right)>0$, which was assumed above. 


\subsection{Update rule for $d \underline{\varepsilon}_{o l}^{i, k}$}

In Step 5 of Algorithm 1, the aim is to find in each integration point an inelastic strain update $d \underline{\varepsilon}_{o l}^{i, k}$, which is done in a similar procedure as described in Sections 4.1 and 4.2. As in Section 3.4, the two-dimensional case is considered only. The intermediate stress quantity $\underline{\boldsymbol{\sigma}}^{0, k}$ from Eq. (41) can be used again. From a careful inspection of the subsequent considerations, one finds that equivalently the tangential inelastic part of strain update $d \underline{\varepsilon}_{T}^{i, k}$ can be included, setting

$$
\underline{\mathbf{\sigma}}^{0, k}:=\underline{\underline{\mathbf{D}}}\left(\underline{\varepsilon}^{k}-\underline{\boldsymbol{\varepsilon}}^{i, k-1}-d \underline{\boldsymbol{\varepsilon}}_{N}^{i, k}-d \underline{\boldsymbol{\varepsilon}}_{T}^{i, k}\right) .
$$

Both settings of $\underline{\boldsymbol{\sigma}}^{0, k}$ will lead to equivalent results. The update direction $\underline{\mathbf{g}}_{o l}^{k}$ is set corresponding to Eq. (34),

$$
\underline{\mathbf{g}}_{o l}^{k}:=\frac{1}{\left\|\boldsymbol{\sigma}_{o l}^{0, k}\right\|}\left[\begin{array}{ccc}
\sigma_{x x}^{0, k} & \frac{1}{2} \sigma_{x y}^{0, k} & 0 \\
\frac{1}{2} \sigma_{x y}^{0, k} & 0 & 0 \\
0 & 0 & 0
\end{array}\right] .
$$

The update $d \underline{\varepsilon}_{o l}^{i, k}$ is chosen as a multiple of the update direction $\underline{\mathbf{g}}_{o l}^{k}$,

$$
d \underline{\varepsilon}_{o l}^{i, k}:=\lambda_{o l} \underline{\mathbf{g}}_{o l}^{k} .
$$

The choice of $\lambda_{o l}$ has yet to be specified. In case the constraint condition $f_{o l}\left(\underline{\boldsymbol{\sigma}}^{0, k}\right) \leq 0$ is satisfied, $\lambda_{o l}$ is set to zero. Otherwise, it is chosen such that the linearized constraint condition

$$
f_{o l}^{*}\left(\underline{\boldsymbol{\sigma}}^{0, k}\right)+\frac{\partial f_{o l}^{*}}{\partial \underline{\boldsymbol{\sigma}}}\left(\underline{\boldsymbol{\sigma}}^{0, k}\right) \cdot\left(\underline{\boldsymbol{\sigma}}^{k}-\underline{\boldsymbol{\sigma}}^{0, k}\right)=0 .
$$

is satisfied, where for the derivative $f_{o l}$ is replaced by the constraint function $f_{o l}^{*}$ with fixed average compressive stress $\sigma_{z z}^{0}$ from Eq. (28). The choice of $d \underline{\varepsilon}_{o l}^{i, k}$ according to Eq. (48) leads to

$$
f_{o l}\left(\underline{\boldsymbol{\sigma}}^{0, k}\right)-\lambda_{o l} \frac{\partial f_{o l}^{*}}{\partial \underline{\boldsymbol{\sigma}}}\left(\underline{\boldsymbol{\sigma}}^{0, k}\right):\left(\underline{\underline{\mathbf{D}}} \underline{\mathrm{g}}_{o l}^{k}\right)=0 .
$$

Inserting definitions of $f_{o l}^{*}$ from Eq. (28) and $\underline{\mathbf{g}}_{o l}^{k}$ from Eq. (47), one obtains $\lambda_{o l}$ by straightforward algebraic manipulations:

$$
\lambda_{o l}=\left\|\boldsymbol{\sigma}_{o l}^{0, k}\right\|^{2} \frac{f_{o l}\left(\underline{\boldsymbol{\sigma}}^{0, k}\right)}{D_{x x x x}\left(\sigma_{x x}^{0, k}\right)^{2}+D_{x y x y}\left(\sigma_{x y}^{0, k}\right)^{2}} .
$$

This value is well-defined as long as $\left\|\boldsymbol{\sigma}_{o l}^{0, k}\right\| \neq 0$, which is always the case if the constraint condition $f_{o l}\left(\underline{\boldsymbol{\sigma}}^{0, k}\right) \leq 0$ is not satisfied.

\section{Examples}

The examples provided in this section are chosen such that they show the correctness of the macroscopic material law, as the obtained results coincide 
with analytical values up to a great accuracy. Thus, geometry and setup of the model are highly simplified. The interested reader is referred to $[18,26]$ for computational results in two and three spatial dimensions, where also comparisons to full-scale finite element contact models have been conducted. In the current paper, no comparative results to such full-scale models are provided, since convergence of the highly nonlinear frictional contact problem could not be obtained for discretizations using a sufficient number of contacting sheets.

The model setup is of very simple geometry: Two piles of layered sheets are connected in one region of mutual overlapping. Together, the two piles form a block of $300 \times 100 \times 100 \mathrm{~mm}$, the length of the overlaps is assumed as $20 \mathrm{~mm}$. The overlaps are arranged in two different manners: first, they are aligned orthogonal to the axial $\mathbf{e}_{x}$-direction, while in a second case, the overlapping region is distorted, such that the sheets are no longer rectangular but trapezoidal. These setups are sketched in Figure 7.

In both settings, a constant, distributed surface load $p_{N}$ is applied to the top surface of the two piles. The vertical $z$-displacement is fixed at the bottom surface, other bearings are described separately for the different load cases. The block is made of steel, with a Young's modulus of $E=2 \cdot 10^{5} \mathrm{~N} / \mathrm{mm}^{2}$, and a Poisson ratio $\nu=0.3$. To simplify analytical considerations, gravity effects are not included in the model. The piles are connected such that they have a total number of $N_{o l}=10$ interfaces, which leads to an overlap density $\rho_{o l}=N_{o l} / h_{o l}=0.1$. The friction coefficient of the surface of the sheets is set to $\mu=0.2$. The three sub-regions of the homogenized block are equally distributed discretized, see Figure 7 . Height and width of the block are discretized by the same number of elements $n_{y}=n_{z}$. The total number of elements in $x$ direction consists $2 \cdot n_{x}$ elements in the left and right pile, plus $n_{x, o l}$ elements along the length of the frictional connection in the center. For the distorted overlapping region, the elements are distorted accordingly. Linear hexahedral finite elements are used in each of the three sub-regions of the homogenized block. All implementations have been done in the framework of the research software HOTINT [27].

\subsection{Orthogonal overlapping connection}

In the sequel, two piles of rectangular sheets are connected in one region of mutual overlapping, as described above. Thus, the overlaps are aligned orthogonal to the axial $\mathbf{e}_{x}$-direction. Three different load cases are considered: Relative axial and transverse displacements applied to the piles, as well as an applied moment.

\subsubsection{Load case 1: Applied axial force}

The first experiment is concerned with pulling apart the two piles in axial $x$-direction of the block. Thus, the $x$-displacement of the first pile is fixed on its left hand side, while a given, constant displacement is prescribed on the right hand side of the second pile. The $y$-component of the displacement is fixed in one single node to eliminate rigid body motions. The setup is displayed in 
Figure 8. The vertical pressure is chosen as $p_{N}=0.25 \mathrm{~N} / \mathrm{mm}^{2}$. Then, the contact normal stress $\sigma_{z z}$ is constant in the whole block, and of value $\sigma_{z z}=p_{N}$. By integration one obtains the total compressive force acting in the overlapping region

$$
F_{N}=p_{N} \cdot l_{o l} \cdot w_{o l}=500 \mathrm{~N}
$$

Inserting this value into Eq. (23) leads to an expected pull force

$$
F_{o l}=\mu \cdot N_{o l} \cdot F_{N}=1000 \mathrm{~N} \text {. }
$$

In computations, the in-plane pull force was measured as the force inflicted by the prescribed displacement on the right end of the block. One expects a linear relation of axial displacement and measured force while the in-plane force is smaller than the limit force $F_{o l}$, and a stagnation of the pull force at a constant level afterwards.

The block was discretized by different numbers of finite elements $n_{x}, n_{y}, n_{z}$ and $n_{x, o l}$. Due to the special setup leading to constant stresses, already a discretization using three finite elements only, i.e. $n_{x}=n_{y}=n_{z}=n_{x, o l}=1$, led to accurate results, which are presented below. Small differences to analytic values arise from chosen error tolerances in the nonlinear iterations.

Figure 9 shows the measured pull force in the computational experiment. In the simulation, a force of $F_{\text {pull }}=1.0011 \mathrm{kN}$ was found, which means a relative error of $0.11 \%$ compared to the analytical value of $1 \mathrm{kN}$. The graphic shows that the measured force coincides with the pull force of an elastic block before sliding starts, and then stays constant at the expected value.

\subsubsection{Load case 2: Applied transversal force}

The second example is concerned with the behavior of the overlapping material block in case of a force acting in transverse direction of the overlaps. To this end, the first pile is fixed on the front surface, while a constant $y$-displacement is prescribed on the front surface of the second pile. A corresponding setup is displayed in Figure 10. Such a configuration leads to inelastic shear stresses in the overlapping region. The necessary force to achieve a relative displacement of the blocks is computed in the same way as in the first load case of axial pulling, leading to a value of $F_{o l}=1 \mathrm{kN}$ for a distributed top load $p_{N}=0.25 \mathrm{~N} / \mathrm{mm}^{2}$. A very coarse discretization with $n_{x}=n_{y}=n_{z}=3$ and $n_{x, o l}=1$ is employed successfully.

In Figure 11, the measured force is displayed as a function of the applied displacement. As in the case of axial pulling, a linear relationship between displacement and force can be seen in the elastic regime while the measured force is below the limit value $F_{o l}$. Afterwards, the pull force stagnates at this constant value. The error measured in computations is approximately $0.03 \%$. The gradual transition from the behavior of an elastic block to the analytical sliding is due to the fact that the sliding starts earlier near the prescribed $y$ displacement. When the whole block is moving, the value of the analytic sliding is reached. 


\subsubsection{Load case 3: Applied moment}

In the third load case, the moment necessary to turn the piles relatively shall be determined. To this end, again an axial $x$-displacement is prescribed on the right hand side of the second pile, while the first pile is fixed on its left hand side as done in load case 1 . In the current load case, the prescribed $x$-displacement is not constant, but linear in the transverse coordinate $y$. Thus, it results in an applied moment, and a stress distribution linear in $y$ for an elastic block. A corresponding model setup is shown in Figure 12.

Again, analytical considerations are employed to verify the behavior of the macroscopic material law. Relative sliding of the blocks occurs whenever the stress $\sigma_{x x}$ reaches the critical value of $\sigma_{x x}^{\max }=\mu \rho_{o l} l_{o l}\left|\sigma_{z z}\right|$. In the current example, the stress is independent of the spatial coordinates $x$ and $z$, but linear in $y$, as is visualized in Figure 13(b). Thus, the relative sliding motion of the two piles will not start at once as in load case 1 where the stress was constant, but a continuous shift from elastic to inelastic behavior is expected. The sliding motion will start for the stress distribution (see Figure 13(b))

$$
\sigma_{x x}(y)=\sigma_{x x}^{\max } \cdot \frac{2 y}{w_{o l}} \quad y \in\left[-\frac{w_{o l}}{2}, \frac{w_{o l}}{2}\right] .
$$

The interfingering piles slide everywhere for the limiting stress distribution where the absolute value of the stress $\sigma_{x x}$ reaches the maximum $\sigma_{x x}^{\max }$ everywhere (see Figure 13(d))

$$
\sigma_{x x}(y)=\operatorname{sign}(y) \sigma_{x x}^{\max } \quad y \in\left[-\frac{w_{o l}}{2}, \frac{w_{o l}}{2}\right] .
$$

An evolution of the stress distribution for increasing applied displacements is depicted in Figure 13(a-d).

In computations, the distributed top load $p_{N}=0.015 \mathrm{~N} / \mathrm{mm}^{2}$ was used. Then, $\sigma_{x x}^{\max }=0.006 \mathrm{~N} / \mathrm{mm}^{2}$. For this case, the applied moment respecting the stress distribution from Eq. (54) under which the block starts sliding is given by

$$
\begin{aligned}
M_{1} & :=\int_{0}^{h_{o l}} \int_{-w_{o l} / 2}^{w_{o l} / 2} \sigma_{x x}(y) y d y d z \\
& =\left.100 \cdot \sigma_{x x}^{\max } \cdot \frac{2}{100} \frac{y^{3}}{3}\right|_{y=-50} ^{y=50}=1000 \mathrm{Nm} .
\end{aligned}
$$

Total sliding and a stagnation of the moment is expected for the moment corresponding to the stress distribution from Eq. (55)

$$
\begin{aligned}
M_{2} & :=\int_{0}^{h_{o l}} \int_{-w_{o l} / 2}^{w_{o l} / 2} \sigma_{x x}(y) y d y d z \\
& =\left.100 \cdot \sigma_{x x}^{\max } \cdot y^{2}\right|_{y=0} ^{y=50}=1500 \mathrm{Nm} .
\end{aligned}
$$

In computations, different discretizations of the model block were used. As the expected stress distributions are linear, for this load case a sufficiently small 
mesh size is needed to obtain good results. Figure 14 displays the measured moment for a discretization with $n_{x}=n_{y}=n_{z}=6$, where the frictional connection is resolved by $n_{x, o l}=3$ elements along its length. The first sliding motion is expected at $M_{1}=1 \mathrm{kNm}$, and total sliding is expected at $M_{2}=$ $1.5 \mathrm{kNm}$. This moment was measured at $M_{2}=1.4965$. The overall accordance with the analytical values is good.

\subsection{Distorted overlapping connection}

Additionally, piles of non-rectangular sheets connected frictionally by mutual overlapping were considered, as depicted in Figure 7(b). To account for the nonrectangular nature of the sheets, a local coordinate system $\left(\mathbf{e}_{x}^{*}, \mathbf{e}_{y}^{*}, \mathbf{e}_{z}^{*}\right)$ had to be used. Different angle were chosen for the direction of the overlaps. The numerical results were satisfying, analytical values for the maximum admissible in-plane pull forces were reproduced by the numerical methods with similar accuracy as was obtained for the rectangular sheets independent of the chosen angle, where the same numbers of elements were used. In the following, results are provided in detail only for the case of axial pulling (load case 1).

\subsubsection{Load case 1: Axial pulling}

The setup is similar to load case 1 for the orthogonal overlaps given above. Bearings and the compressive distributed load $p_{N}=0.25 \mathrm{~N} / \mathrm{mm}^{2}$ are kept at the original level. The necessary pull force $F_{o l}$ is computed in the same way as above, leading to a value of $F_{o l}=1 \mathrm{kN}$.

Again, the force necessary to pull the stack apart is measured. Figure 15 shows the measured force as a function of the applied axial $x$-displacement. The behavior is equivalent to the results obtained for the orthogonal overlaps, as is expected. The maximal measured pull force lies at $1.0001 \mathrm{kN}$, which results in a relative error of approximately $0.01 \%$.

\section{Conclusion}

A homogenized material model for the computational simulation of thinsheet piles has been presented in previous works of the authors $[1,2]$. The application of this material law to large piles leads to a significant reduction of unknowns and thereby to an according decrease of CPU-time compared to fullscale contact computations. The main contribution of this work is the extension of this material to regions, where different piles interact by mutual overlapping. Phenomenological considerations lead to constraints applied to in-plane stresses, which are treated similar to yield functions known from elasto-plasticity. A numerical procedure for the algorithmic treatment of the nonlinear macroscopic material law is provided. The material behavior was verified by computations for model problems where the simulation results could be compared to analytical values. These analytical values were reached with high accuracy even for coarse discretizations. The experiments imply that the proposed homogenization is feasible also for more complicated problem setups, as known from practical applications, e.g. iron cores of large power transformers. 


\section{Acknowledgements}

The present research has been performed in collaboration with Siemens Transformers Austria Linz GmbH \& Co KG. Support of the authors by a grant of the K2-Comet Austrian Center of Competence in Mechatronics (ACCM) is gratefully acknowledged.

\section{References}

[1] L. G. Vorhauer, J. Gerstmayr, Mechanically homogenized material model for a pack of sheets, Proceedings in Applied Mathematics and Mechanics (PAMM) 9 (1) (2009) 423-424.

[2] L. G. Aigner, J. Gerstmayr, A. S. Pechstein, A two-dimensional homogenized model for a pile of thin elastic sheets with frictional contact, Acta Mechanica (2010) 1-13.

[3] Dassault Systèmes, Simulia, Abaqus Unified FEA, http://www. simulia.com (2008).

[4] S. Nemat-Nasser, M. Hori, Micromechanics: overall properties of heterogeneous materials, Vol. 37 of North-Holland Series in Applied Mathematics and Mechanics, North-Holland Publishing Co., Amsterdam, 1993.

[5] A. E. Omri, A. Fennan, F. Sidoroff, A. Hihi, Elastic-plastic homogenization of layered composites, European Journal of Mechanics - A/Solids 19 (4) (2000) 585-601.

[6] C. Pellegrino, U. Galvanetto, B. Schrefler, Numerical homogenization of periodic composite materials with non-linear material components, International Journal for Numerical Methods in Engineering 46 (10) (1999) 16091637 .

[7] P. Ladevéze, G. Lubineau, D. Marsal, Towards a bridge between the microand mesomechanics of delamination for laminated composites, Composites Science and Technology 66 (6) (2006) 698-712.

[8] F. Greco, Homogenized mechanical behavior of composite micro-structures including micro-cracking and contact evolution, Engineering Fracture Mechanics 76 (2) (2009) 182-208.

[9] E. Sacco, A nonlinear homogenization procedure for periodic masonry, European Journal of Mechanics - A/Solids 28 (2) (2009) 209 - 222.

[10] J. Heyman, The stone skeleton, Internat. J. Solids and Structures 2 (1966) 249-279.

[11] G. D. Piero, Constitutive equation and compatibility of the external loads for linear elastic masonry-like materials, Meccanica 24. 
[12] M. Angelillo, Constitutive relations for no-tension materials, Meccanica 28 (3) (1993) 195-202.

[13] G. Milani, P. Lourenco, A. Tralli, Homogenixed limit analysis of masonry walls, part I: Failure surfaces, Computers \& Structures 84 (3-4) (2006) 166-180.

[14] G. Milani, P. Lourenco, A. Tralli, Homogenixed limit analysis of masonry walls, part II: Structural examples, Computers \& Structures 84 (3-4) (2006) 181-195.

[15] S. D. Pasquale, New trends in the analysis of masonry structures, Meccanica 27 (3) (1992) 173-184.

[16] M. Lucchesi, M. Silhav, N. Zani, Equilibrated divergence measure stress tensor fields for heavy masonry bodies, European Journal of Mechanics A/Solids 28 (2) (2009) $223-232$.

[17] P. Wriggers, Computational Contact Mechanics, 2nd Edition, Springer, Berlin, Heidelberg, New York, 2006.

[18] L. Aigner, A. Pechstein, J. Gerstmayr, H. Irschik, Stability of thin sheet layered structures with frictional contact, in: Proceedings of the IV European Conference on Computational Mechanics (Solids, Structures and Coupled Problems in Engineering), Paris, France, May 16-21, 2010.

[19] J. Gerstmayr, A. Pechstein, L. Aigner, A three-dimensional continuous material model for a pile of thin sheets and frictional contact, in: Proceedings of the Tenth International Conference on Computational Structures Technology, B.H.V. Topping et Al. (Eds.), Civil-Comp Press, Scotland, Valencia, Spain, Sept. 2010, 2010.

[20] T. A. Laursen, Computational Contact and Impact Mechanics, Springer, New York, 2002.

[21] F. Ziegler, Mechanics of Solids and Fluids, Springer, Berlin, 1995.

[22] J. C. Simo, T. J. R. Hughes, Computational Inelasticity, Vol. 7 of Interdisciplinary Applied Mathematics: Mechanics and Materials, Springer, New York Berlin Heidelberg, 1998.

[23] E. H. Dill, Continuum mechanics: elasticity, plasticity, viscoelasticity, CRC Press, Boca Raton, 2007.

[24] H. Mang, G. Hofstetter, Festigkeitslehre, Springer, Wien, 2000.

[25] D. Mohr, Mechanism-based multi-surface plasticity model for ideal truss lattice materials, International Journal of Solids and Structures 42 (2005) $3235-3260$. 
[26] L. Aigner, A. Sinwel, J. Gerstmayr, H. Irschik, The constitutive modeling of homogenized contact and friction conditions in thin-sheet packages, Proceedings of the 7th EUROMECH Solid Mechanics Conference, Lisbon, Portugal.

[27] J. Gerstmayr, HOTINT - a C++ environment for the simulation of multibody dynamics systems and finite elements, in: K. Arczewski, J. Fraczek, M. Wojtyra (Eds.), Proceedings of the Multibody Dynamics 2009 Eccomas Thematic Conference, 2009, pp. 1-20. 
FIGURE 1: Model problem consisting of two piles connected by one overlapping region. The local coordinate system $\left(\mathbf{e}_{x}^{*}, \mathbf{e}_{y}^{*}, \mathbf{e}_{z}^{*}\right)$, length $l_{o l}$, width $w_{o l}$, and height $h_{o l}$ of the overlapping region are depicted.

FIGURE 2: Left: Relative sliding motion for two piles. Right: Corresponding continuous deformation in a homogenized overlapping region.

FIGURE 3: (a) For one single interface, the norm of the in-plane force $\left\|F_{o l}\right\|$ needs to be smaller or equal $\mu\left|F_{N}\right|$; (b) for multiple interfaces, the term $\mu\left|F_{N}\right|$ is multiplied by the number of interfaces $N_{o l}$.

FIGURE 4: Hexahedral of infinitesimal width $d y$, height $d z$, spanning the whole overlap length $l_{o l}$ in $x$-direction: (a) microscopic view including single sheets, (b) homogenized equivalent macroscopic view with averaged compressive force $F_{N}$.

FIGURE 5: Microscopic view: relative displacement for a constant stress vector $\boldsymbol{\sigma}_{o l}$. Relative displacement $\dot{\mathbf{u}}^{r}$ and $\boldsymbol{\sigma}_{o l}$ point into the same direction.

FIGURE 6: Macroscopic view: homogenized model of the setup shown in Figure 4, using the "inelastic" displacement $\dot{\mathbf{u}}^{i}$ of the two stacks. The "inelastic" displacement gradient $\dot{\mathbf{u}}_{x}^{i}$ with respect to normal direction and $\boldsymbol{\sigma}_{o l}$ point into the same direction. The "inelastic" displacement gradient $\dot{\mathbf{u}}_{, y}^{i}$ with respect to transverse direction vanishes.

FIGURE 7: Different setups for the model problem: Two piles are connected in one region of mutual overlapping, which is (a) orthogonal to the axial $x$-direction, (b) distorted at a certain angle. The block is discretized using $n_{x}, n_{y}, n_{z}$ and $n_{x, o l}$ elements in the different directions.

FIGURE 8: Model setup for load case 1 (applied axial force).

FIGURE 9: Axial force necessary for a given $x$-displacement of an elastic block and a block of the macroscopic material including an overlapping region.

FIGURE 10: Model setup for load case 2 (applied transversal force).

FIGURE 11: graphics/transverseforce Transversal force necessary for a given $y$-displacement of an elastic block and a block of the macroscopic material including an overlapping region.

FIGURE 12: Model setup for load case 3 (applied moment).

FIGURE 13: Evolution of the stress distribution in the overlapping region for an increasing linear prescribed displacement. (a) no sliding, (b) marks the distribution where sliding starts, (c) sliding in parts of the overlapping region, (d) limiting case: the piles move relatively in the whole overlapping region.

FIGURE 14: Moment necessary for a given $x$-displacement of an elastic block and a block of the macroscopic material including an overlapping region.

FIGURE 15: Axial force necessary for a given $x$-displacement of an elastic block and a block of the macroscopic material including an overlapping region. Different angles of the overlapping region were chosen. 

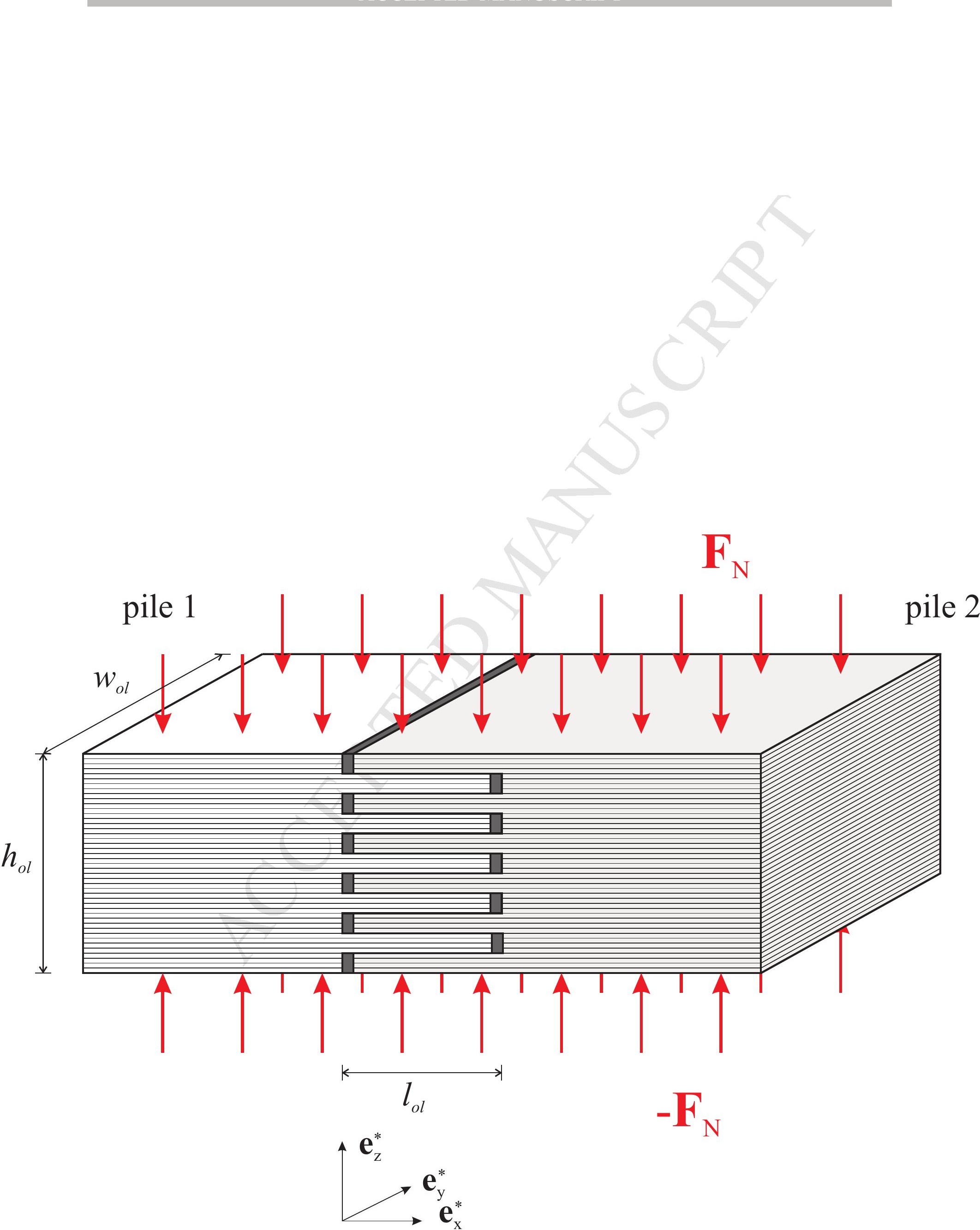

$\mathbf{F}_{\mathrm{N}}$ 


\begin{abstract}
)
\end{abstract}

$$
\stackrel{\mathbf{e}_{\mathrm{z}}^{*}}{\longrightarrow} \mathbf{e}_{\mathrm{y}}^{*}
$$

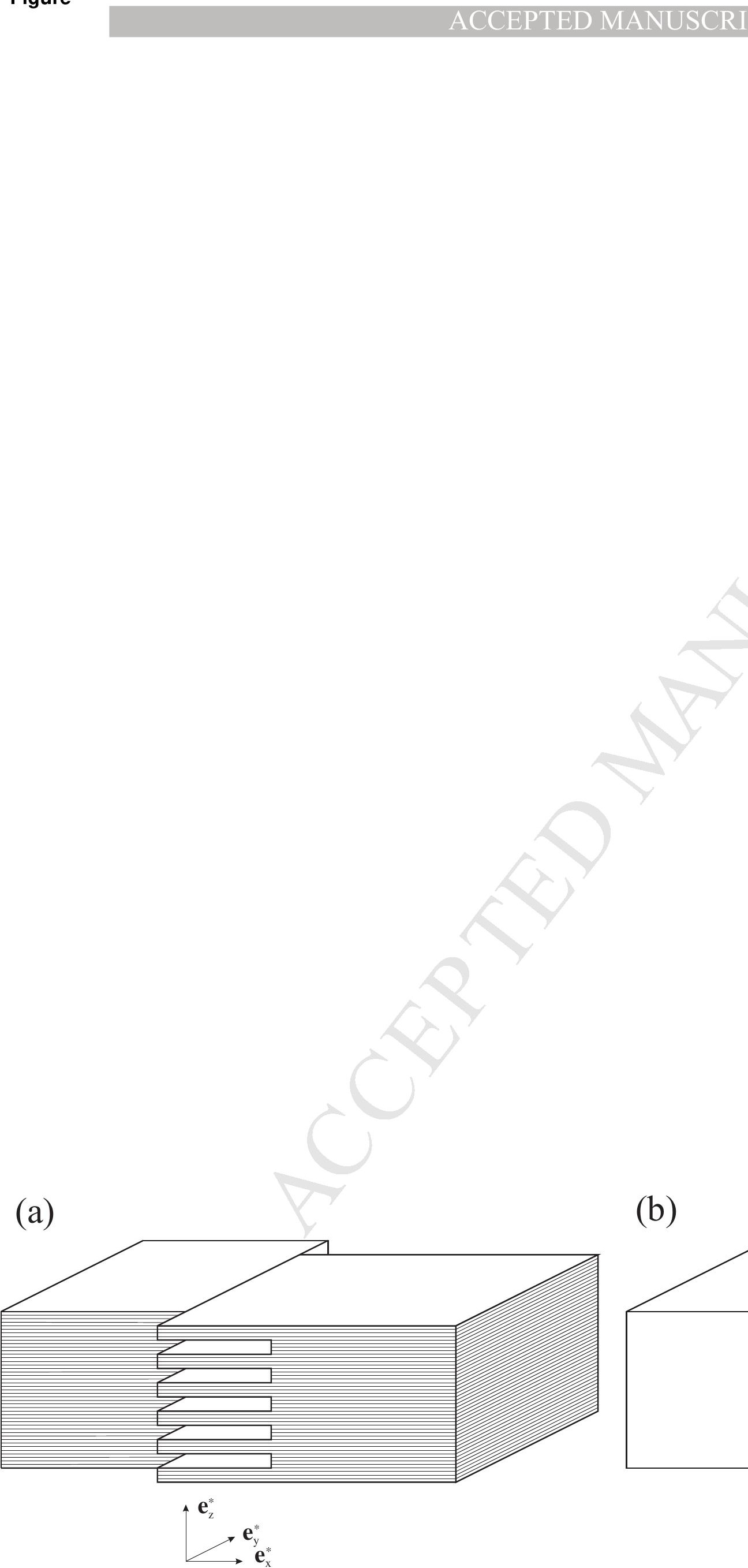

(b)

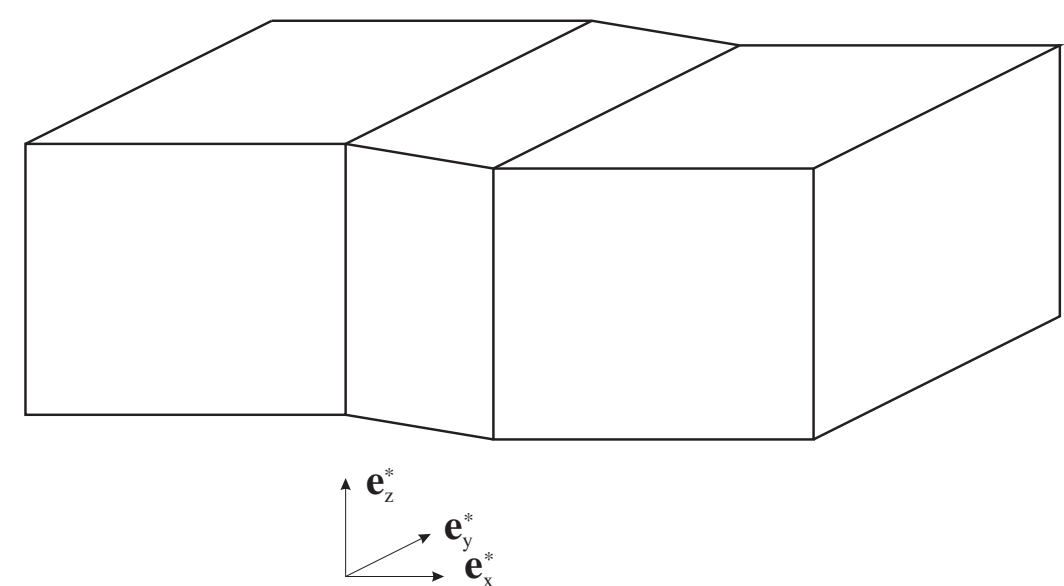

(a)

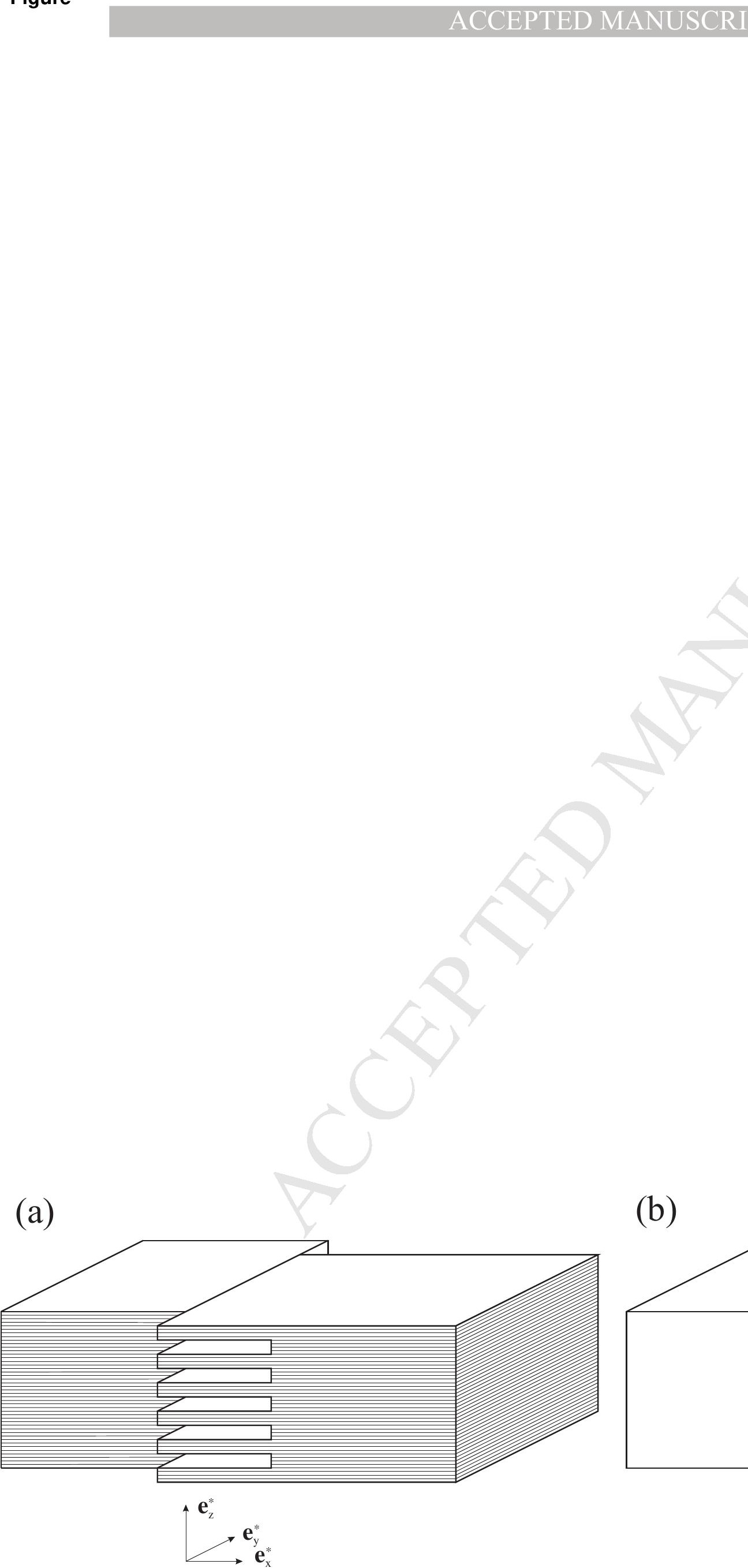




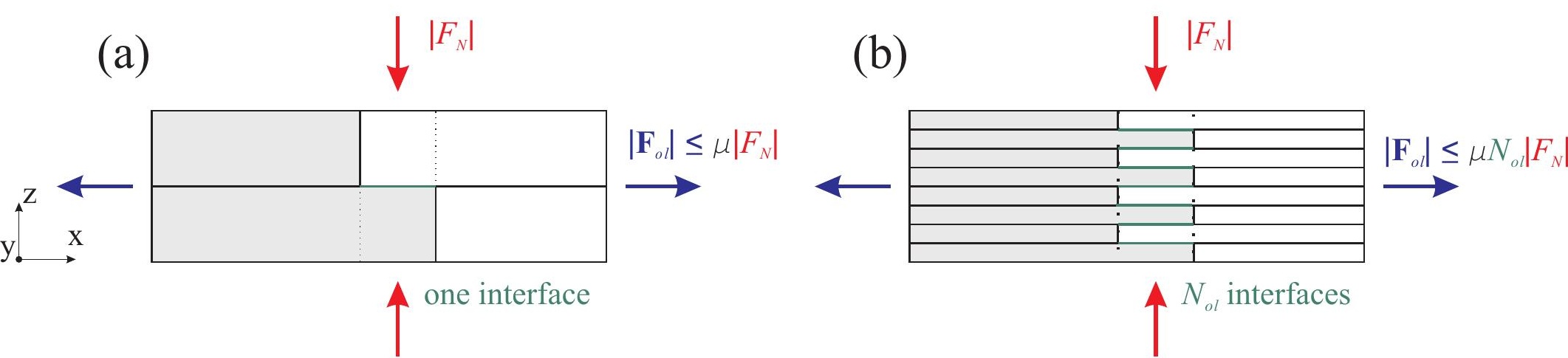




\section{$\mathbf{u}^{r} \sim \boldsymbol{\sigma}_{o l}$}

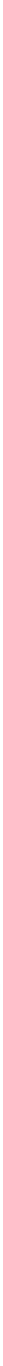

Figure 

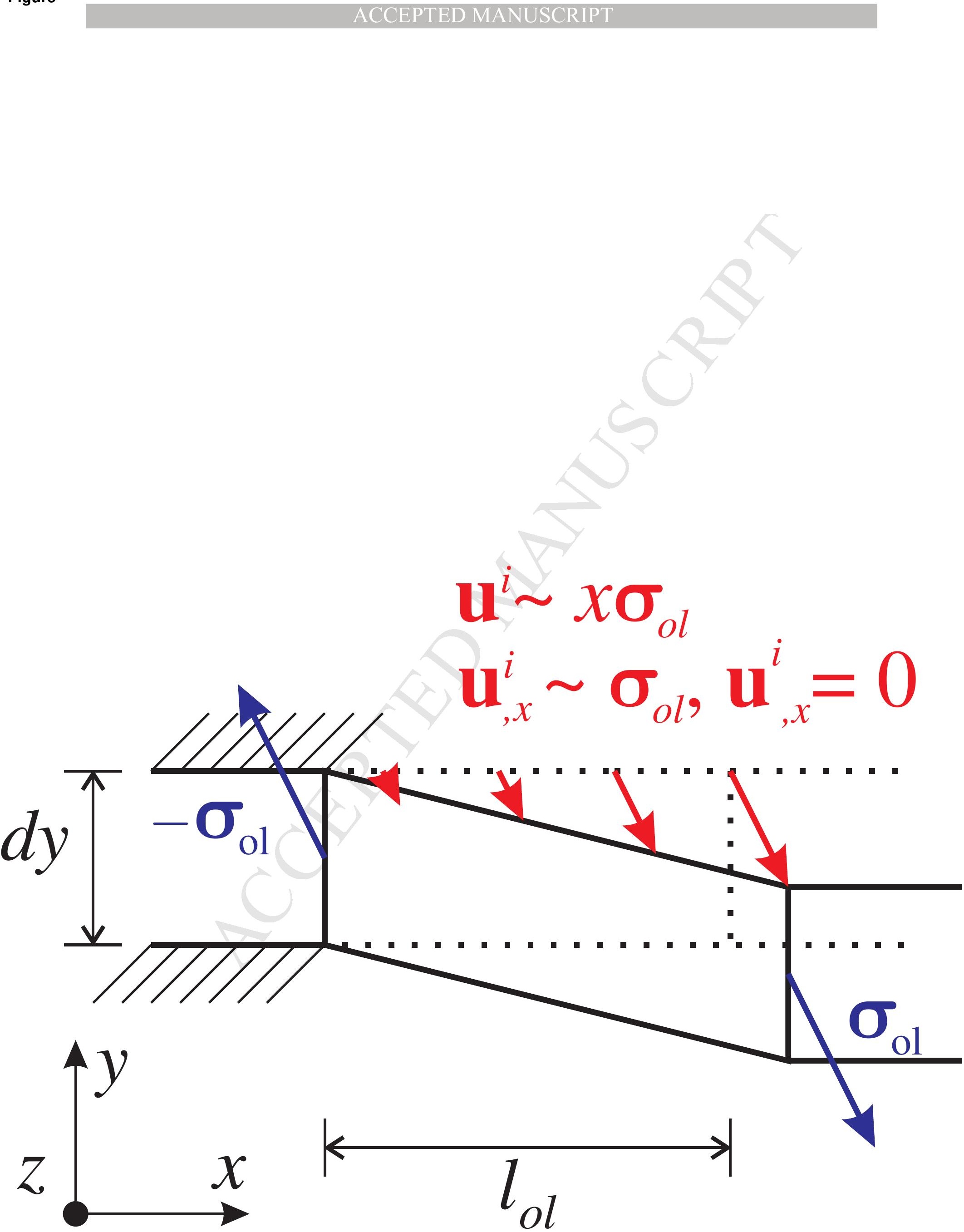

$\mathbf{u}_{, x}^{i} \sim \boldsymbol{\sigma}_{o l}, \mathbf{u}_{, x}^{i}=0$ 


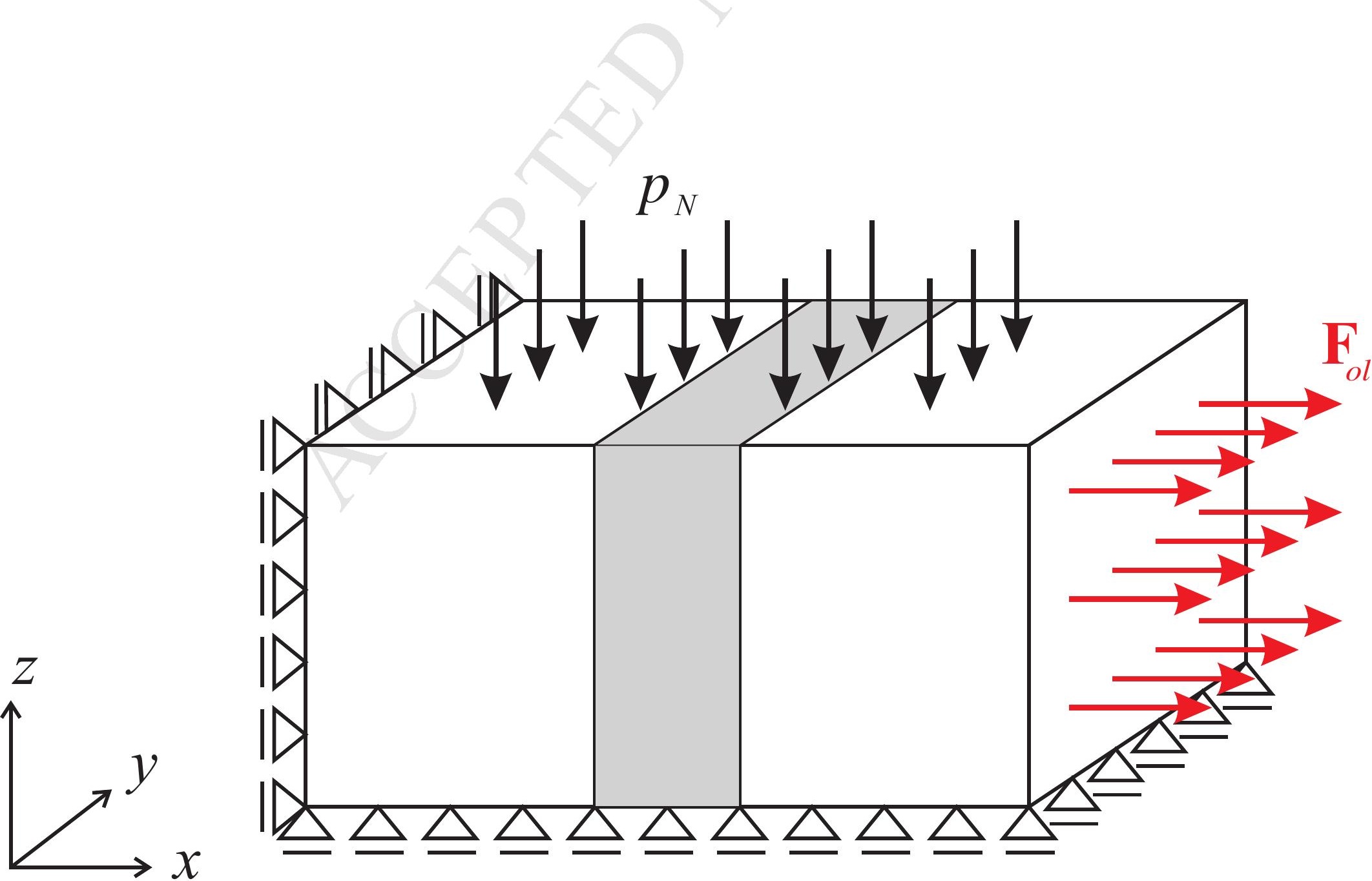

$z$

个

.

Figure 


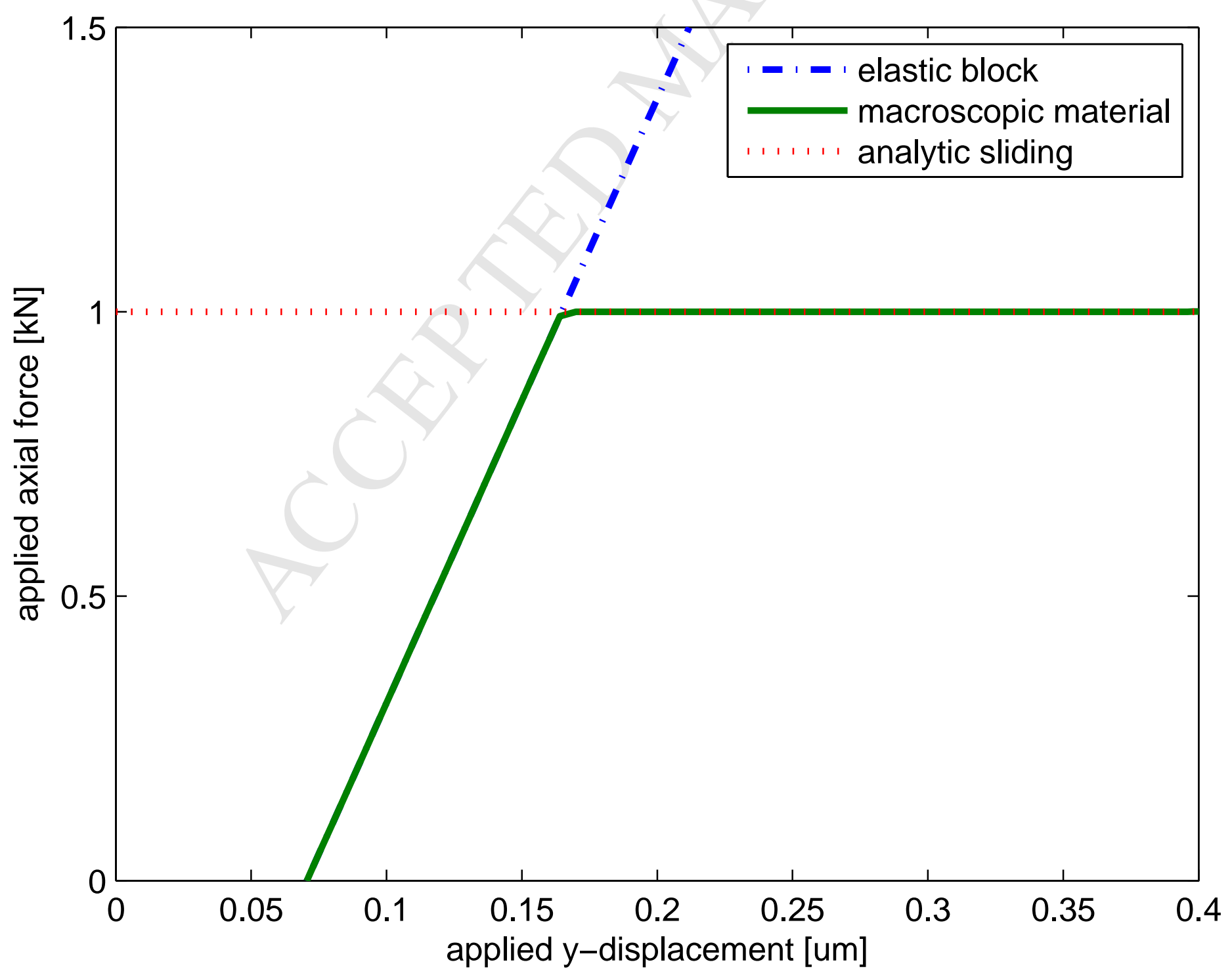


$z$
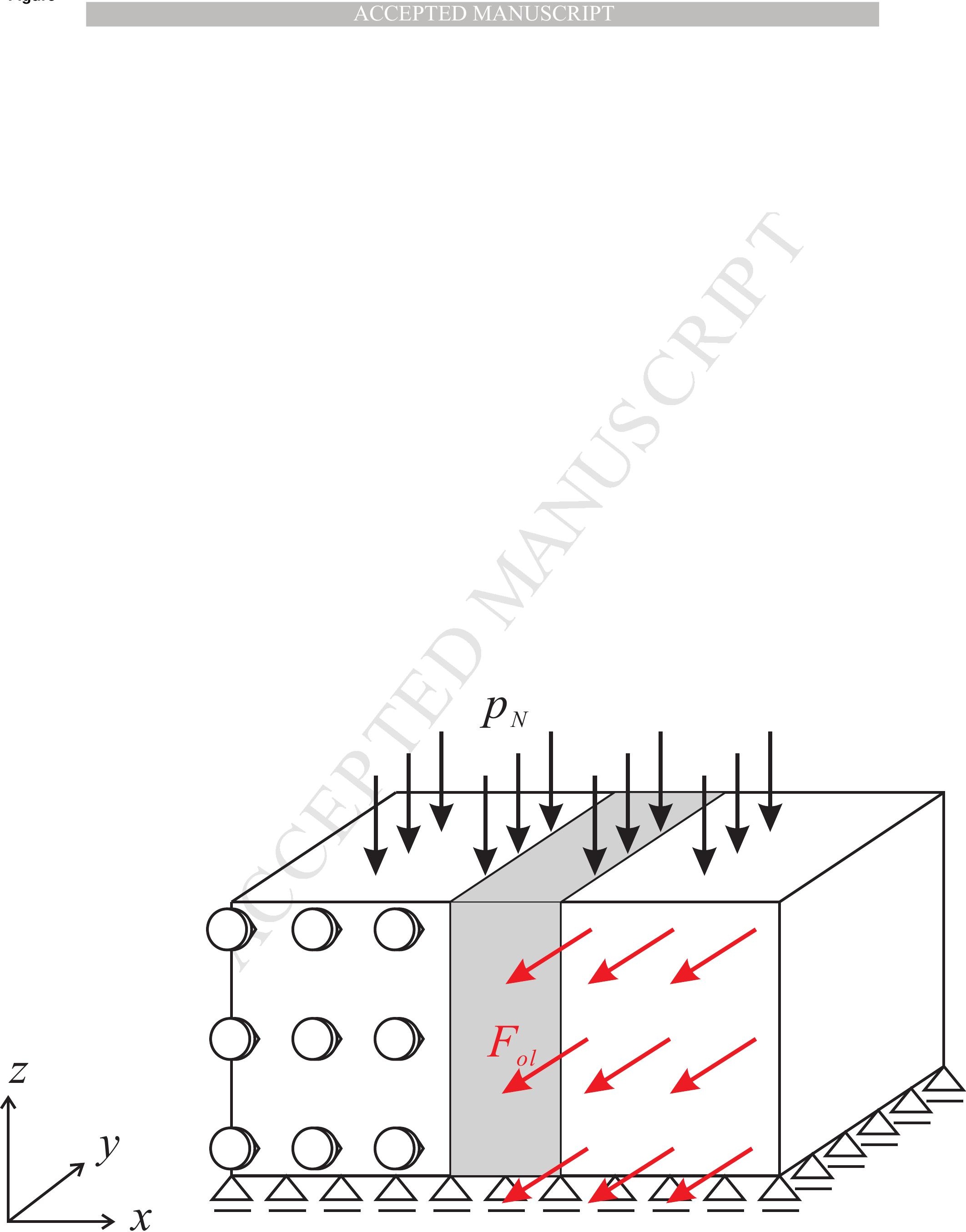


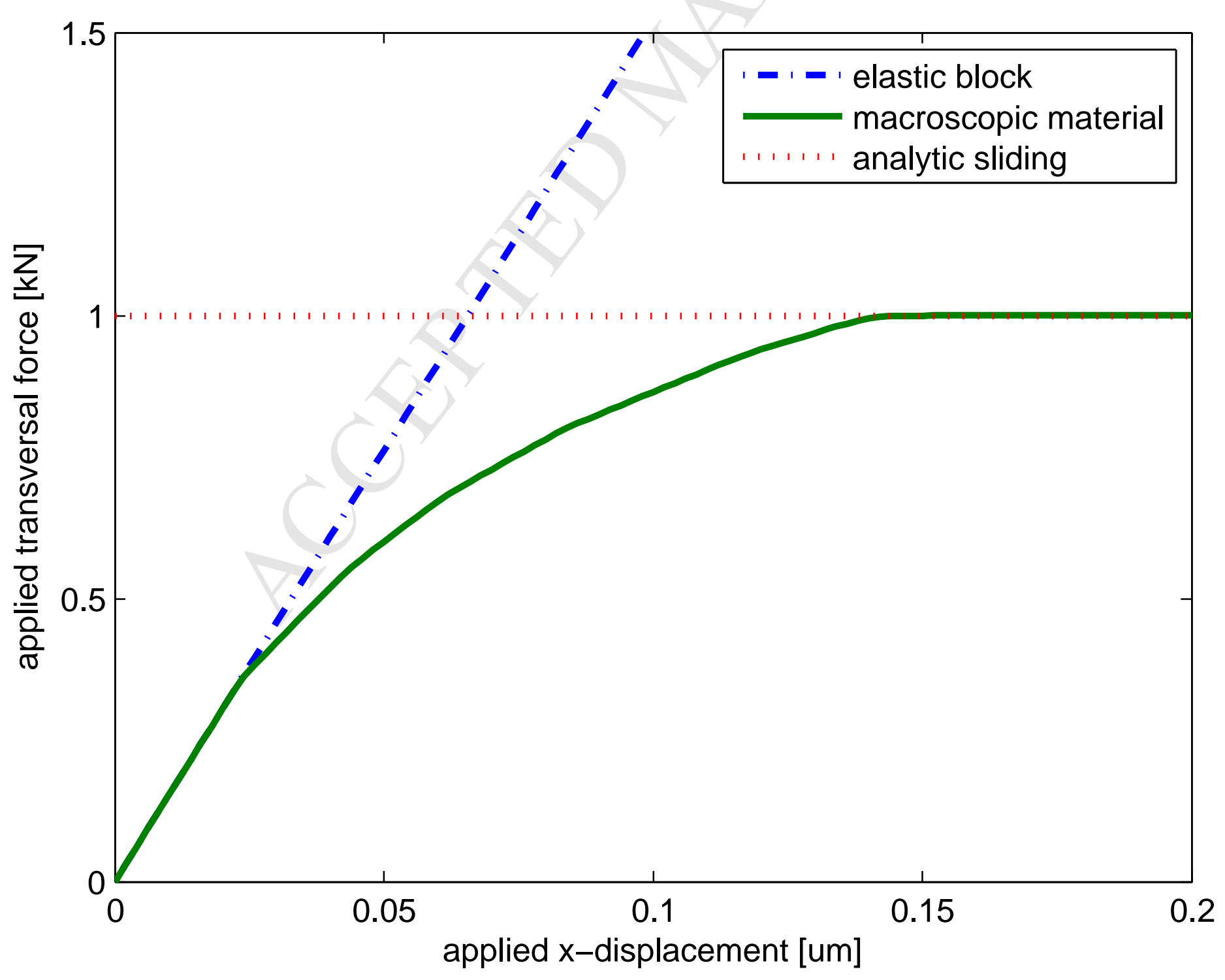



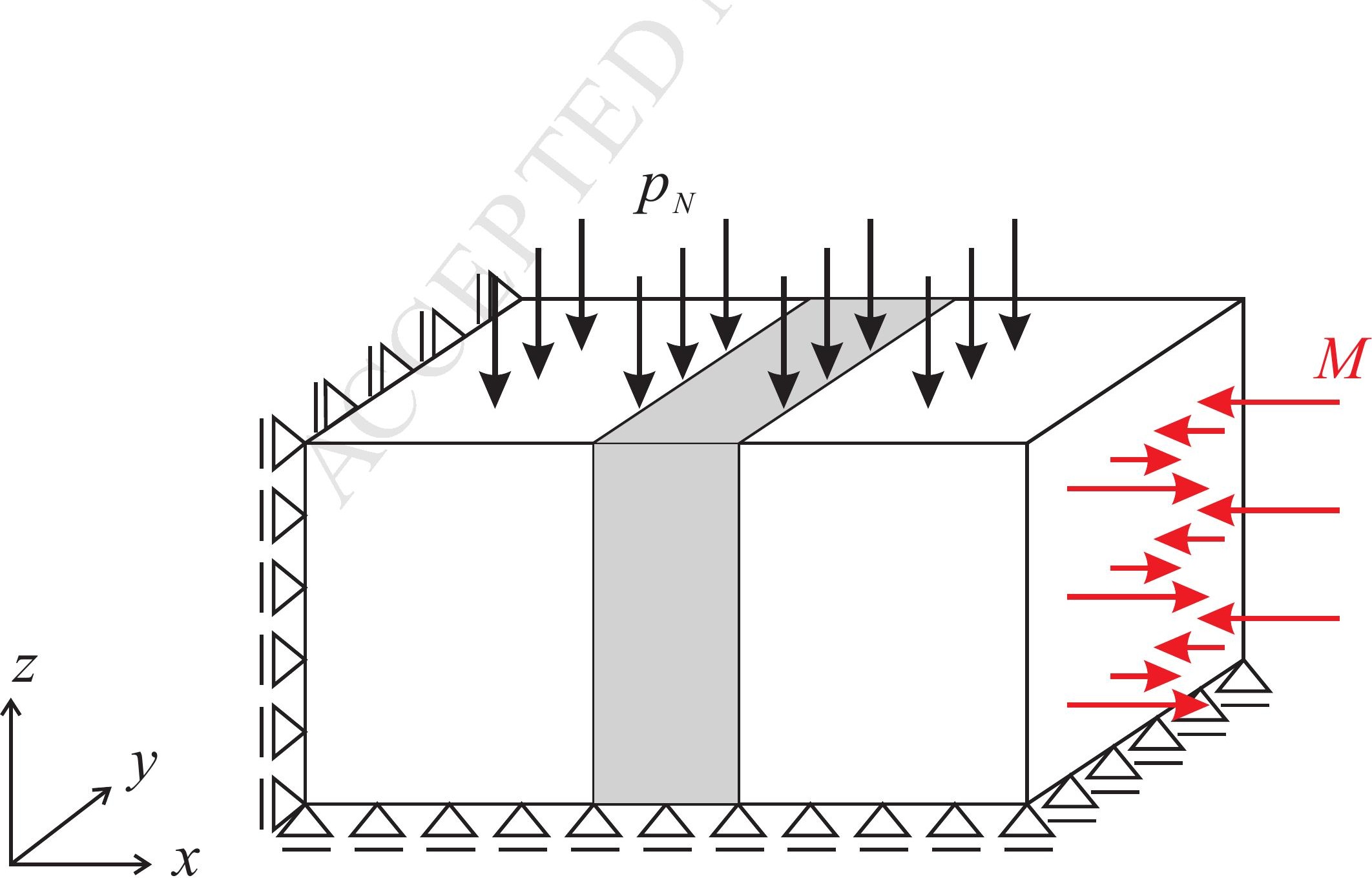

Figure 
(a)

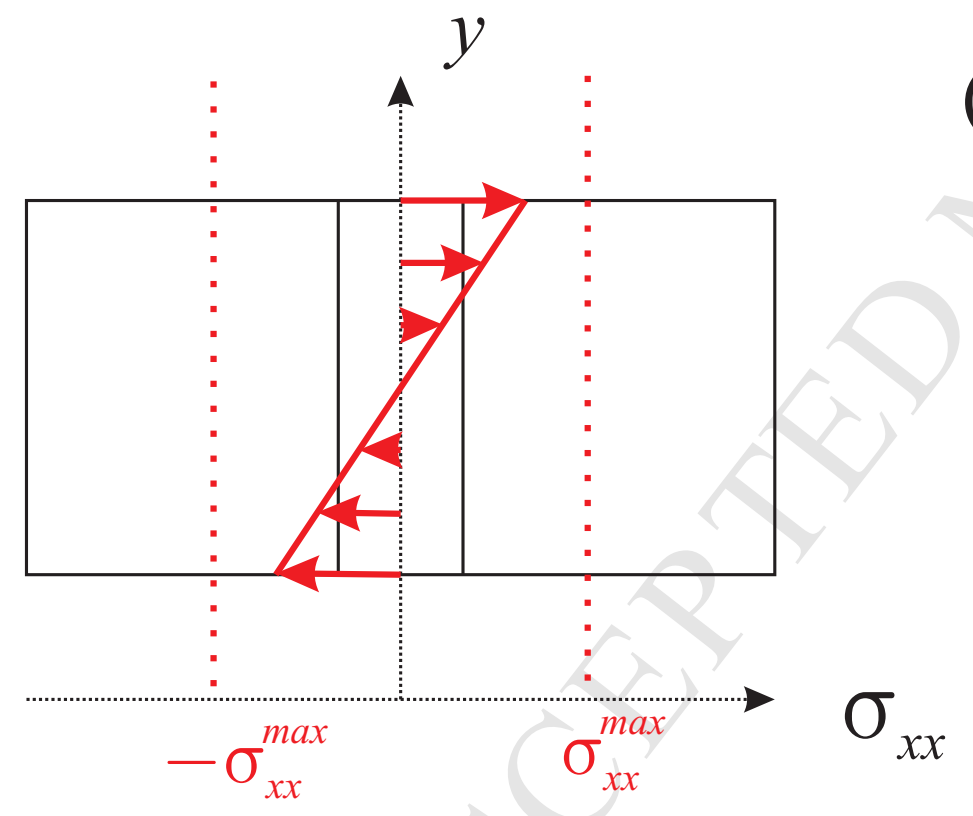

(c)

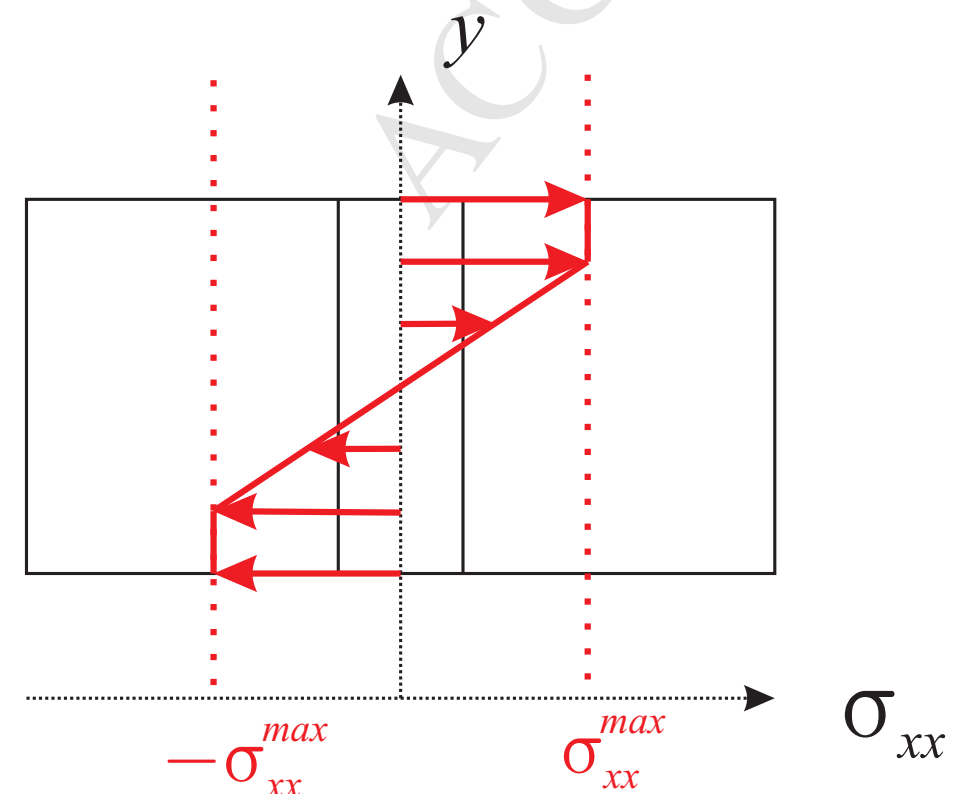

(b)
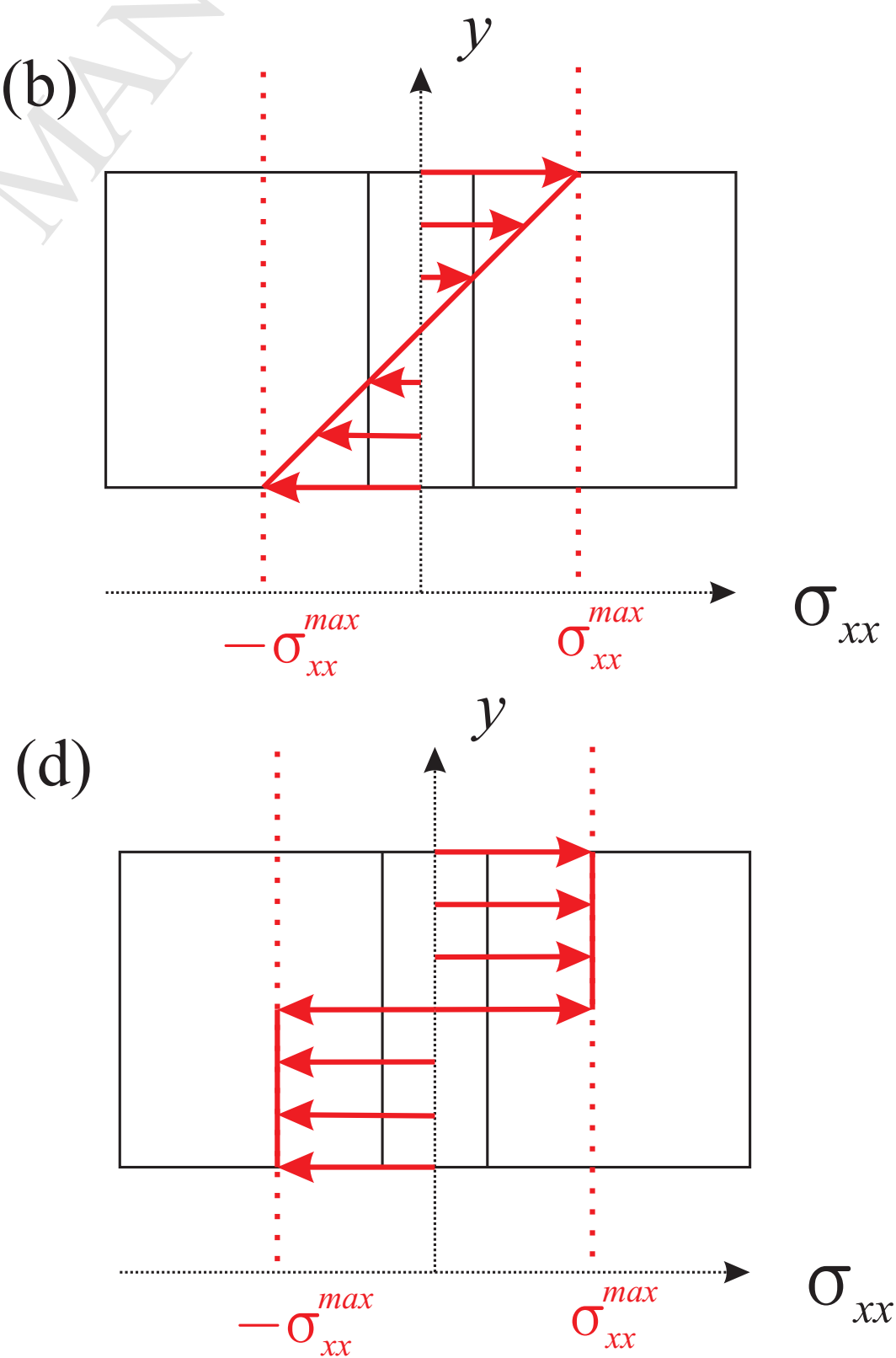


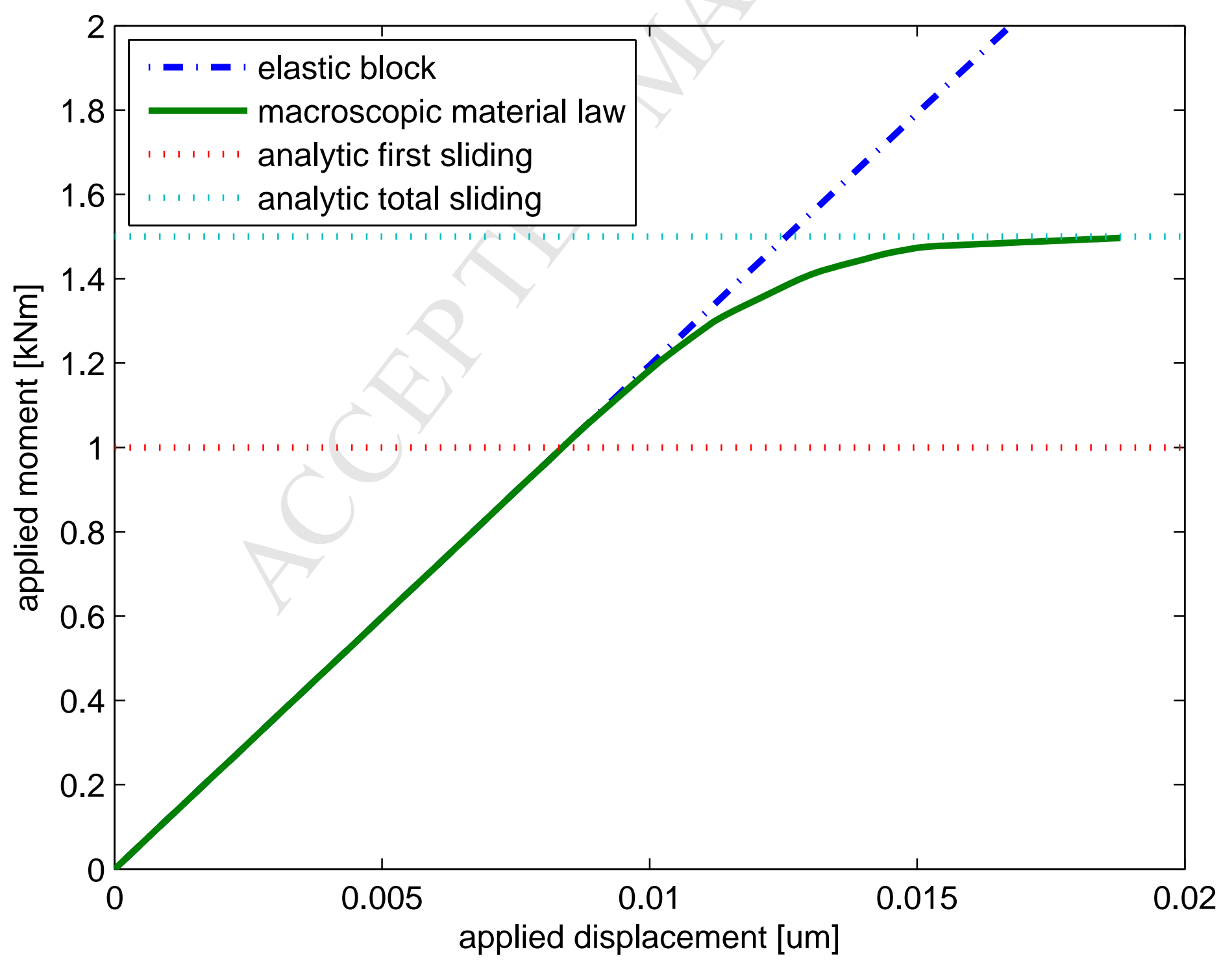




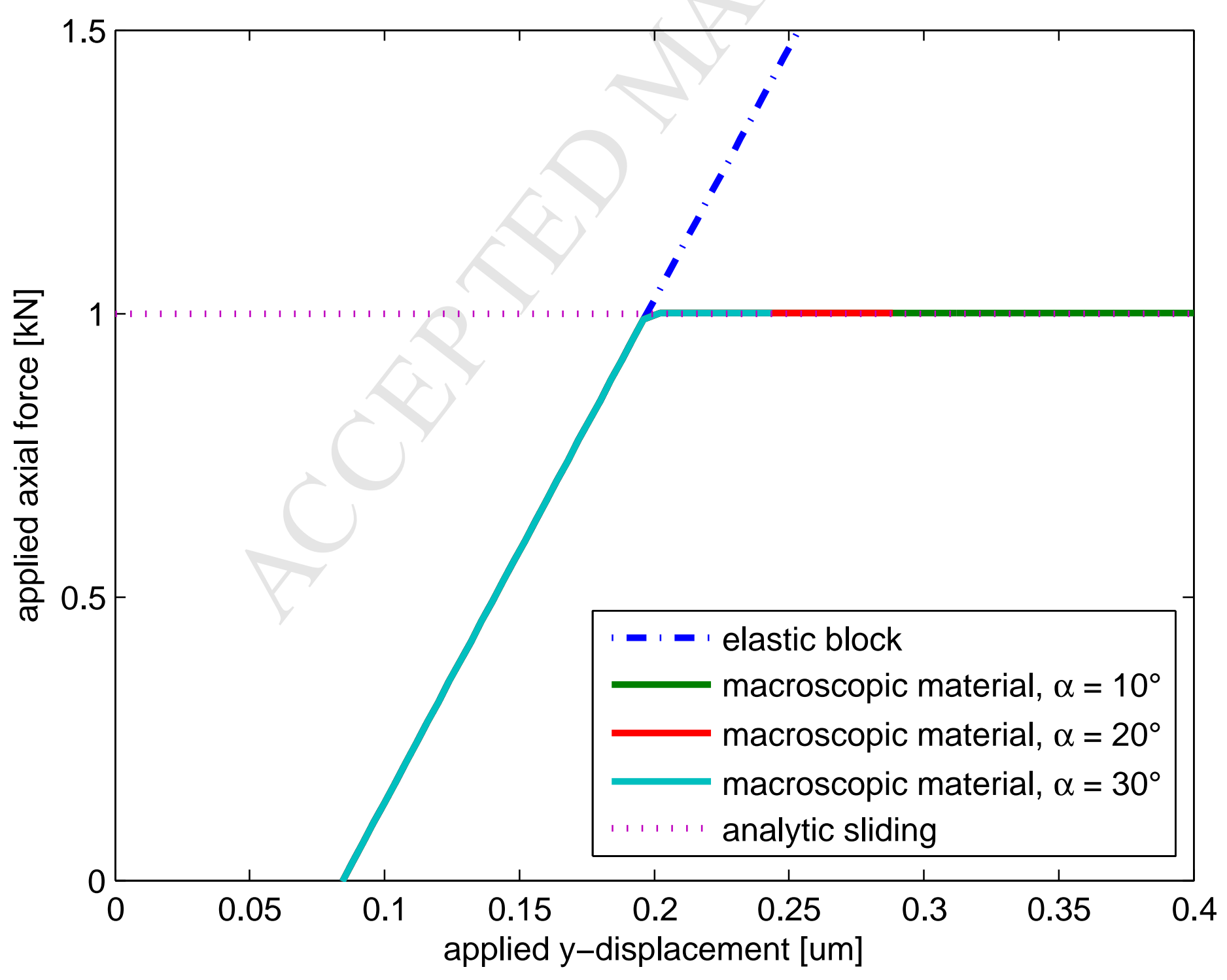

